\title{
Linking globular cluster structural parameters and their evolution: multiple stellar populations
}

\author{
Alessandra Mastrobuono-Battisti ${ }^{\oplus 1,2 \star}$ and Hagai B. Perets ${ }^{\oplus 3,4}$ \\ ${ }^{1}$ Department of Astronomy and Theoretical Physics, Lund Observatory, Box 43, SE-221 00 Lund, Sweden \\ ${ }^{2}$ Max Planck Institute for Astronomy, Königstuhl 17, D-69117 Heidelberg, Germany \\ ${ }^{3}$ Physics Department, Technion - Israel Institute of Technology, Haifa 3200003, Israel \\ ${ }^{4}$ Cahill Center for Astrophysics, Division of Physics, Mathematics and Astronomy, California Institute of Technology, Pasadena, CA 91125, USA
}

Accepted 2021 May 12. Received 2021 May 12; in original form 2020 November 20

\begin{abstract}
Globular clusters (GCs) are known to host multiple stellar populations showing chemical anomalies in the content of light elements. The origin of such anomalies observed in Galactic GCs is still debated. Here we analyse data compiled from the Hubble Space Telescope, ground-based surveys, and Gaia Data Release 2 (DR2) and explore relationships between the structural properties of GCs and the fraction of second population (2P) stars. Given the correlations we find, we conclude that the main factor driving the formation/evolution of $2 \mathrm{P}$ stars is the cluster mass. The existing strong correlations between the $2 \mathrm{P}$ fraction and the rotational velocity and concentration parameter could derive from their correlation with the cluster mass. Furthermore, we observe that increasing cluster escape velocity corresponds to higher $2 \mathrm{P}$ fractions. Each of the correlations found is bimodal, with a different behaviour detected for low- and high-mass (or escape velocity) clusters. These correlations could be consistent with an initial formation of more centrally concentrated 2P stars in deeper cluster potentials, followed by a long-term tidal stripping of stars from clusters outskirts. The latter are dominated by the more extended distributed first population (1P) stars, and therefore stronger tidal stripping would preferentially deplete the $1 \mathrm{P}$ population, raising the cluster $2 \mathrm{P}$ fraction. This also suggests a tighter distribution of initial $2 \mathrm{P}$ fractions than observed today. In addition, higher escape velocities allow better retention of low-velocity material ejected from $1 \mathrm{P}$ stars, providing an alternative/additional origin for the observed differences and the distributions of $2 \mathrm{P}$ fractions amongst GCs.
\end{abstract}

Key words: globular clusters: general-Galaxy: kinematics and dynamics - Galaxy: stellar content.

\section{INTRODUCTION}

For decades globular clusters (GCs) have been thought to represent the perfect example of simple stellar populations. All the stars in each GC were thought to have formed concurrently and with the same chemical composition. Since leftover gas could be ejected due to supernovae winds, no further star formation episodes were considered possible. However, photometric and spectroscopic studies collected over the last decade (see e.g. Piotto et al. 2007; Carretta et al. 2009) have progressively revealed a different picture, showing that most, if not all, Galactic GCs host multiple stellar populations with different light elements content. While the first, pristine, stellar population has a chemical composition similar to halo field stars with similar metallicity, the stars belonging to the second stellar population present numerous chemical anomalies, including anticorrelations between the content of different elements (e.g. C-N, Na-O, and in some cases $\mathrm{Mg}-\mathrm{Al}$; Gratton, Carretta \& Bragaglia 2012; Gratton et al. 2019). These anomalies seem to be ubiquitous, pointing toward a possible common origin of multiple populations in all Galactic GCs. Similar anomalies have been observed in stellar clusters of different ages in the Large Magellanic Cloud (LMC) and Small Magellanic

^E-mail: mastrobuono@astro.lu.se
Cloud (SMC), making the puzzle even more complex to solve (see Bastian \& Lardo 2018, for a review on the topic).

Several scenarios have been proposed to explain the existence of the observed multiple populations, but no clear solution has been yet found (see Renzini et al. 2015; Bastian \& Lardo 2018; Gratton et al. 2019, for a summary of the scenarios and for their caveats). One possibility is that GCs experienced two or more star formation episodes, in which second population (2P) stars formed from processed gas lost by massive first population (1P) stars, and/or accreted external gas.

In the so-called self-enrichment scenarios, in which the gas is provided by $1 \mathrm{P}$ stars, the nature of the possible donors is constrained by the chemical properties of the $2 \mathrm{P}$ stars. Different potential sources of the gas have been identified [e.g. asymptotic giant branch (AGB) stars, fast-rotating massive stars; Ventura et al. 2001; Decressin et al. 2007; Breen 2018]. Alternative scenarios predict that the anomalous stars form as a consequence of processes that can only happen in primordial GCs (e.g. accretion of processed material on to the zero-age main-sequence stellar disc, formation and evolution of a central supermassive star; Bastian et al. 2013; Gieles et al. 2018; Wang et al. 2020).

The later dynamical evolution of GCs can affect the properties of the GCs and their 1P/2P stars on longer time-scales, potentially leaving observable signatures on the internal structure of the cluster. 

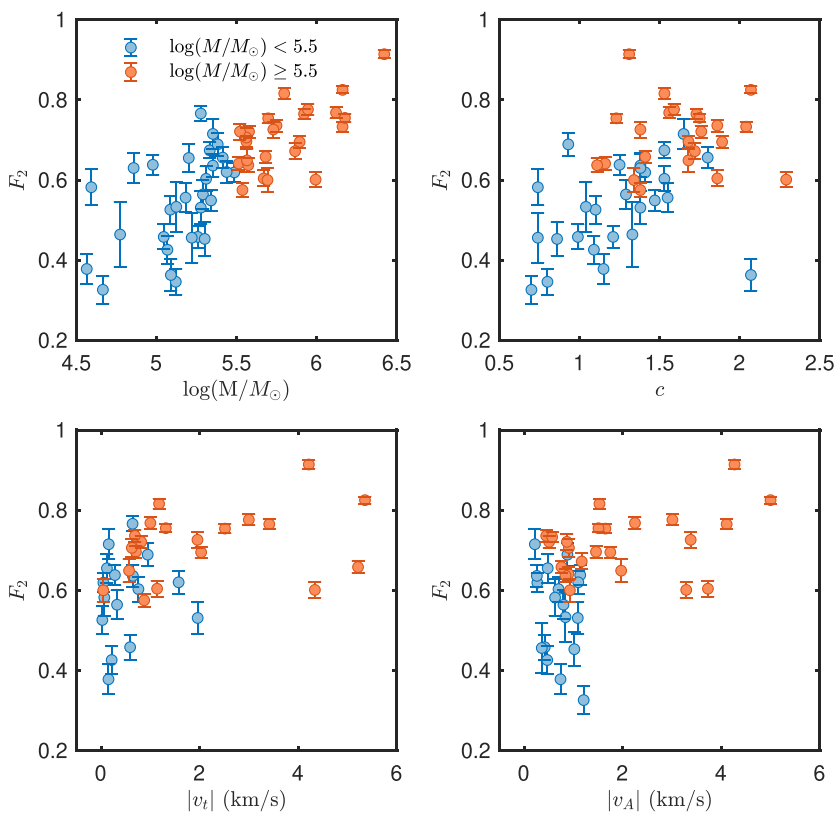

Figure 1. The second generation number fraction, $F_{2}$, for the available cluster sample is plotted against different GC internal parameters. From topleft to bottom-right, $F_{2}$ is plotted against the logarithm of the current cluster mass, the cluster concentration, the rotational velocity of the clusters on the plane of the sky, and the 3D rotational velocity. Orange symbols are clusters with masses larger than $10^{5.5} \mathrm{M}_{\odot}$, and light blue symbols are clusters with masses smaller than the same threshold.

The amplitude of the leftover signatures will depend on the amount of 2P stars that form compared to the $1 \mathrm{P}$ stars, leading to relationships between internal cluster properties and the fraction of $2 \mathrm{P}$ stars.

For example, the self-enrichment scenarios predict that the $2 \mathrm{P}$ stars form centrally concentrated. According to these models, the higher concentration should be still observed today in clusters that are not fully relaxed (Mastrobuono-Battisti \& Perets 2013, 2016; Vesperini et al. 2013, 2021; Hénault-Brunet et al. 2015; Tiongco, Vesperini \& Varri 2019). Centrally concentrated 2P stars have been indeed observed in a number of clusters (see e.g. Norris \& Freeman 1979; Milone et al. 2012a; Richer et al. 2013; Cordero et al. 2014, 2017; Bellini et al. 2015; Dalessandro et al. 2019; Kamann et al. 2020; Dondoglio et al. 2021; Szigeti et al. 2021). The observed differences are milder for more dynamically evolved systems (Dalessandro et al. 2019). Moreover, simulations predict kinematic differences between the two populations, including differential rotation and discrepancies in the velocity dispersions (Mastrobuono-Battisti \& Perets 2013, 2016; Vesperini et al. 2013; Hénault-Brunet et al. 2015; Tiongco et al. 2019). This kind of differences have been observed in Galactic GCs (Richer et al. 2013; Bellini et al. 2015, 2018; Cordero et al. 2017; Lee 2017, 2018; Dalessandro et al. 2018; Milone et al. 2018; Cordoni et al. 2020c; Kamann et al. 2020), however, the strength and variety of the signatures hamper the interpretation of the results.

Carretta et al. (2010) used principal component analysis and multivariate relations to study the phase of transition between $1 \mathrm{P}$ and $2 \mathrm{P}$, finding that the main parameters driving the chemical signatures found in GCs are the metallicity, mass, and cluster age (see also Martocchia et al. 2018, 2019; Lagioia et al. 2019). Carretta (2019) extended these results, identifying the mass and the concentration as the two most important parameters determining the extent of the chemical anomalies observed in Galactic GCs.
More recently, Milone et al. (2020) used the 'chromosome maps' of 59 Galactic GCs and of 11 GCs of both Magellanic Clouds to compare the multiple populations phenomenon in different environments. In this way they found correlations between the $2 \mathrm{P}$ properties and the internal and orbital parameters of the clusters. In particular, the fraction of $1 \mathrm{P}$ stars exhibit a strong anticorrelation with the mass of the $2 \mathrm{P}$ and with the initial cluster mass. They also found a mild correlation between the $2 \mathrm{P}$ and current cluster mass. The $1 \mathrm{P}$ fraction is correlated with the total GC luminosity and clusters with larger perigalactic radii show larger helium variations. In addition, Galactic and extragalactic clusters seem to follow similar relationships.

We note that in self-accreting scenarios, material lost by $1 \mathrm{P}$ stars could be lost if ejected at velocities higher than the escape velocity of the cluster. For example, mass lost through winds of evolved stars typically have velocities of $10-30 \mathrm{~km} \mathrm{~s}^{-1}$, comparable with the escape velocities of clusters. Clusters with low escape velocities might therefore retain only a fraction of the mass lost through winds, while more massive and concentrated clusters with higher escape velocities would retain more. In fact, if the escape velocity from the cluster is higher than the fastest winds from evolved stars, all the mass lost through winds will be retained, and one might expect all clusters above this threshold to have comparable $2 \mathrm{P}$ fractions, independent of their mass.

In this work, we carry out a systematic search of correlations between different internal cluster parameters, complementing previous works and extending them to the analysis of the correlations with other internal properties. We use data collected from the literature to assess the possible relationships existing between cluster internal properties and the fraction of $2 \mathrm{P}$ stars. In Section 2, we introduce the different data sets that we employed in our study. In Section 3, we present the results of our analysis. In Section 4, we discuss the results and draw our conclusions.

\section{DATA SETS AND ANALYSIS}

To study the correlations between internal GC parameters focusing on the presence of $2 \mathrm{P}$ stars, we collected data from different sources. The $2 \mathrm{P}$ number fractions, to which we refer to quantify the $2 \mathrm{P}$ phenomenon, are from Milone et al. (2017). We used the Harris (1996, 2010 edition) catalogue for most of the structural parameters (metallicity, ellipticity, concentration, core radius, and half-mass relaxation time). The masses of the clusters have been obtained from Gnedin \& Ostriker (1997). As a further test, we have also used the masses from Baumgardt \& Hilker (2018). The cluster ages are taken from the most recent homogeneous compilation in the literature, presented by VandenBerg et al. (2013).

Gaia Data Release 2 (DR2) provided detailed information on their radial velocities of stars in the outskirts of clusters and allowed the estimate of the clusters orbital parameters, including the radial period, $T_{r}$ (Gaia Collaboration et al. 2018). Proper motions have a larger coverage of clusters since photometric measures are less affected by the crowding. As an estimate of the 3D rotational velocity, we used the amplitude of the rotation estimated by Sollima, Baumgardt \& Hilker (2019) from Gaia DR2 proper motions and literature line-of-sight velocities of Galactic GCs. We also used the rotation on the plane of the sky evaluated by Bianchini et al. (2018) using the same Gaia DR2 data as another a proxy of the cluster internal rotation.

We quantified the relationships between the internal properties of GCs by evaluating the Pearson correlation coefficients and probabilities. These indicators are, however, strongly affected by the presence of outliers and only check for the presence of linear correlations. 
Table 1. Statistical correlation coefficients (PCC) and probabilities (PP) between $F_{2}$ and several GC internal parameters. The statistical parameters are reported for the entire sample of GCs and for clusters less massive (LM) and more massive (HM) than $10^{5.5} \mathrm{M}_{\odot}$. P stands for Pearson, $\mathrm{S}$ for Spearman, and $\mathrm{K}$ for Kendall.

\begin{tabular}{|c|c|c|c|c|c|c|c|c|}
\hline $\operatorname{Par}_{1}$ & $\mathrm{Par}_{2}$ & Stat & PCC & PP & $\mathrm{PCC}_{\mathrm{LM}}$ & $\mathrm{PP}_{\mathrm{LM}}$ & $\mathrm{PCC}_{\mathrm{HM}}$ & $\mathrm{PP}_{\mathrm{HM}}$ \\
\hline \multirow[t]{3}{*}{$F_{2}$} & \multirow[t]{3}{*}{$M$} & $\mathrm{P}$ & 0.76 & $3.2 \times 10^{-11}$ & 0.50 & 0.0056 & 0.64 & $5.3 \times 10^{-4}$ \\
\hline & & S & 0.77 & $1.5 \times 10^{-11}$ & 0.54 & 0.0023 & 0.62 & $9.5 \times 10^{-4}$ \\
\hline & & K & 0.58 & $4.9 \times 10^{-10}$ & 0.38 & 0.0038 & 0.45 & 0.0020 \\
\hline \multirow[t]{3}{*}{$F_{2}$} & \multirow[t]{3}{*}{$c$} & $\mathrm{P}$ & 0.50 & $2.9 \times 10^{-4}$ & 0.37 & 0.063 & 0.025 & 0.91 \\
\hline & & S & 0.55 & $4.5 \times 10^{-5}$ & 0.48 & 0.013 & 0.10 & 0.64 \\
\hline & & K & 0.40 & $6.2 \times 10^{-5}$ & 0.40 & 0.0092 & 0.56 & 0.73 \\
\hline \multirow{3}{*}{$F_{2}$} & \multirow{3}{*}{$\left|v_{t}\right|$} & $\mathrm{P}$ & 0.46 & 0.0043 & 0.092 & 0.73 & 0.34 & 0.14 \\
\hline & & S & 0.54 & $8.7 \times 10^{-4}$ & 0.19 & 0.47 & 0.41 & 0.071 \\
\hline & & K & 0.38 & $8.0 \times 10^{-4}$ & 0.12 & 0.56 & 0.32 & 0.055 \\
\hline \multirow[t]{3}{*}{$F_{2}$} & \multirow{3}{*}{$\left|v_{A}\right|$} & $\mathrm{P}$ & 0.47 & 0.0016 & -0.25 & 0.32 & 0.38 & 0.066 \\
\hline & & S & 0.43 & 0.0042 & -0.22 & 0.38 & 0.32 & 0.12 \\
\hline & & K & 0.30 & 0.0052 & -0.18 & 0.33 & 0.21 & 0.16 \\
\hline \multirow[t]{3}{*}{$F_{2}$} & \multirow[t]{3}{*}{$V / \sigma$} & $\mathrm{P}$ & 0.28 & 0.11 & 0.12 & 0.67 & 0.13 & 0.60 \\
\hline & & S & 0.33 & 0.056 & -0.014 & 0.96 & 0.21 & 0.38 \\
\hline & & K & 0.21 & 0.083 & -0.017 & 0.96 & 0.17 & 0.34 \\
\hline \multirow[t]{3}{*}{$F_{2}$} & \multirow[t]{3}{*}{$\varepsilon$} & $\mathrm{P}$ & 0.082 & 0.57 & -0.12 & 0.56 & 0.52 & 0.0073 \\
\hline & & S & 0.079 & 0.58 & -0.22 & 0.28 & 0.49 & 0.012 \\
\hline & & K & 0.058 & 0.57 & -0.15 & 0.32 & 0.36 & 0.015 \\
\hline \multirow[t]{3}{*}{$F_{2}$} & \multirow[t]{3}{*}{ Age } & $\mathrm{P}$ & $3.5 \times 10^{-3}$ & 0.98 & 0.24 & 0.23 & -0.28 & 0.21 \\
\hline & & S & -0.049 & 0.74 & 0.25 & 0.22 & -0.36 & 0.10 \\
\hline & & K & -0.039 & 0.71 & 0.18 & 0.22 & -0.28 & 0.081 \\
\hline \multirow[t]{3}{*}{$F_{2}$} & \multirow[t]{3}{*}{$\mathrm{Age} / t_{\mathrm{rh}}$} & $\mathrm{P}$ & -0.31 & 0.031 & -0.15 & 0.44 & -0.33 & 0.13 \\
\hline & & S & -0.16 & 0.26 & 0.14 & 0.48 & -0.37 & 0.092 \\
\hline & & K & -0.11 & 0.27 & 0.10 & 0.47 & -0.25 & 0.11 \\
\hline \multirow[t]{3}{*}{$F_{2}$} & \multirow{3}{*}{$\log \left(t_{\mathrm{rh}}\right)$} & $\mathrm{P}$ & 0.25 & 0.071 & -0.068 & 0.73 & 0.47 & 0.018 \\
\hline & & $S$ & 0.14 & 0.31 & -0.18 & 0.35 & 0.40 & 0.049 \\
\hline & & K & 0.093 & 0.32 & -0.13 & 0.33 & 0.28 & 0.055 \\
\hline \multirow[t]{3}{*}{$F_{2}$} & \multirow[t]{3}{*}{ Age $/ T_{r}$} & $\mathrm{P}$ & $2.7 \times 10^{-3}$ & 0.88 & 0.38 & 0.091 & -0.36 & 0.19 \\
\hline & & S & $3.9 \times 10^{-3}$ & 0.98 & 0.45 & 0.043 & -0.24 & 0.38 \\
\hline & & K & $6.3 \times 10^{-3}$ & 0.97 & 0.34 & 0.031 & -0.16 & 0.44 \\
\hline
\end{tabular}

Hence, we also calculated the Spearman and Kendal coefficients and probabilities, which attribute less weight to the outliers and can detect non-linear correlations. While the coefficients represent the strength and direction of the linear correlation between the two variables taken into account, we consider a probability smaller than the significance level of 0.05 to indicate the rejection of the hypothesis that no correlation exists between the two quantities. All the tests have been run for the whole sample of clusters and for clusters less or more massive than $10^{5.5} \mathrm{M}_{\odot}$ separately. The results of the analysis are presented in the next section.

In Appendix A, the same tests are repeated considering metal-rich $([\mathrm{Fe} / \mathrm{H}]>-1)$ and metal-poor $([\mathrm{Fe} / \mathrm{H}] \leq-1)$ GCs separately, as well as for the entire sample of GCs. In Appendix B, we detail the results obtained using the projected rotational velocity instead of the 3D one. In Appendix C, we discuss the age correlations obtained using different data sets available in literature (Dotter et al. 2010; Forbes \& Bridges 2010; Gratton et al. 2010). In Appendix D, we discuss the existence of a correlation between the second generation fraction and the cluster binary fraction.

\section{RESULTS}

Currently, none of the proposed formation scenarios are able to reproduce all the chemical signatures observed for the stars belonging to the 2P in each cluster (Renzini et al. 2015; Bastian \& Lardo 2018; Gratton et al. 2019). In the following, we present the resulting relations between the cluster structural and kinematic properties and the fractions of $2 \mathrm{P}$ stars. The parameter space that needs to be explored is extremely large. Therefore, we restricted our search to the most promising structural parameters, like the cluster masses, ages, rotation, and concentrations. As these results can come from existing correlations between the $2 \mathrm{P}$ fraction and the internal properties of the clusters, they might shed light on the formation scenario.

For the first time, we conduct a systematic search that considers all the relevant structural parameters for all the observed Galactic GCs and that adopts the second generation number fraction as the only reference to quantify the $2 \mathrm{P}$ phenomenon.

\subsection{Multiple populations and their major correlations with cluster properties}

Fig. 1 shows the fraction of second generation stars, $F_{2}$ (quantity available for $\sim 1 / 3$ of the Galactic GCs; Milone et al. 2017), as a function of different Galactic GC internal parameters. In this figure, we present the strongest correlations found, while the full information on all the statistical parameters can be found in Table 1 .

\subsubsection{GC masses}

As already found by Milone et al. $(2017,2020)$, we confirm that $F_{2}$ increases with the total mass of the clusters (top left-hand panel of Fig. 1). In our analysis we adopted the masses given by Gnedin \& 

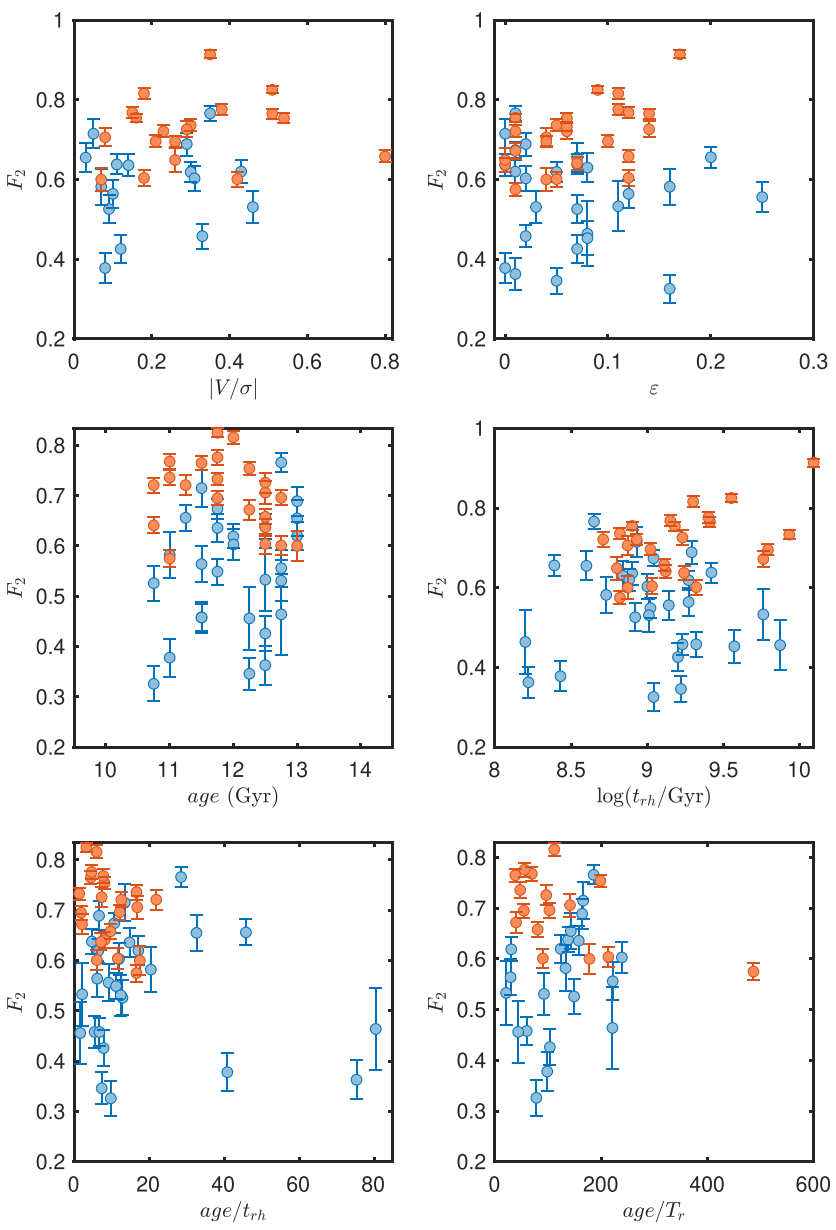

Figure 2. The second generation stellar number fraction, $F_{2}$, is plotted against the cluster $|V / \sigma|$ parameter (top left-hand panel), the cluster ellipticity (top right-hand panel), the cluster age (middle left-hand panel), the logarithm of the half-mass relaxation time (middle right-hand panel), the dynamical age (bottom left-hand panel), and the age over period (bottom right-hand panel). Orange symbols are clusters with masses larger than $10^{5.5} \mathrm{M}_{\odot}$, and light blue symbols are clusters with masses smaller than the same threshold.

Ostriker (1997). However, we obtain similar results when using the more recent mass compilation provided by Baumgardt \& Hilker (2018). As shown by the coefficients listed in Table 1, this correlation is tight (the correlation coefficient ranges between 0.58 and 0.77 , depending on the statistical test being performed). In self-enrichment scenarios, more massive clusters can provide and retain more material for an additional star formation event, leading naturally to a positive correlation between the mass and $F_{2}$. For masses smaller than $10^{5.5} \mathrm{M}_{\odot}$ the relationship shows a significant scatter and becomes bimodal. The four main outliers (NGC 6366, NGC 2298, NGC 4833, and NGC 6681) show a larger $F_{2}$ compared to other clusters of similar mass. Those clusters are significantly dynamically evolved clusters; in particular, NGC 6366 and NGC 2298 are heavily stripped clusters (de Marchi \& Pulone 2007; Paust et al. 2009), the orbit of NGC 4833 suggests that bulge shocking removed a large fraction of the initial mass of this cluster (Carretta et al. 2014), and NGC 6681 is a corecollapsed cluster (Harris 1996). Moreover, the perigalactic distance of these clusters is relatively small (Gaia Collaboration et al. 2018), confirming that they could have lost a significant fraction of their original stellar population residing in the cluster outskirts due to the tidal interaction with the Galaxy (see also Milone et al. 2020, for a study of the correlation between $F_{2}$ and the perigalactic distance of the clusters). If $2 \mathrm{P}$ stars form in the cluster's core, GCs that lost a large fraction of their initial mass are expected to have larger $F_{2}$. In this scenario, the less concentrated $1 \mathrm{P}$ was, indeed, more easily stripped from the outskirts of the cluster because of evaporation and tidal interaction with the Galactic potential.

\subsubsection{Concentration parameter}

$F_{2}$ strongly correlates with the concentration parameter-an indicator of the depth of the central potential of the cluster (see top right-hand panel of Fig. 1). The correlation exists for the whole sample of GCs and for the clusters less massive than $10^{5.5} \mathrm{M}_{\odot}$. If $2 \mathrm{P}$ stars form in the cluster core, this population will be more bound in more concentrated clusters, and therefore less affected by tidal effects than $1 \mathrm{P}$ stars. While low-mass clusters cover a large range of $F_{2}$, massive clusters, which can retain a large fraction of gas, have $2 \mathrm{P}$ fractions that only vary between 0.6 and 0.8 . The two main outliers that show higher concentration than expected, NGC 6717 and NGC 7078, are both core-collapsed clusters. Therefore, their concentration parameters are not accurate and should be considered only as indicative (Harris 1996). The main outlier showing a concentration smaller than expected is NGC 5139 ( $\omega$ Cen), which is strongly suspected to be the former nucleus of an accreted and destroyed dwarf galaxy (Norris \& Da Costa 1995).

\subsubsection{GC rotations}

While Bianchini et al. (2018) used Gaia data to provide the rotation signal of clusters on the plane of the sky $\left(v_{t}\right)$, Sollima et al. (2019) combined Gaia proper motions to the most comprehensive set of line-of-sight velocities of Galactic GCs to obtain their 3D rotational amplitudes $\left(v_{A}\right)$. We plotted $F_{2}$ against both these quantities, finding a positive correlation (see bottom panels of Fig. 1). The large scatter and bimodality observed when using $v_{t}$ is reduced when plotting $F_{2}$ against $v_{A}$. The non-rotating clusters, i.e. the ones showing rotational signal close to zero, show in both cases a large scatter. The scatter is reduced for clusters with higher rotational velocities. A relationship between $F_{2}$ and the rotational velocity is expected if $2 \mathrm{P}$ stars form from gas lost by $1 \mathrm{P}$ stars in a rotating cluster (see e.g. Hénault-Brunet et al. 2015; Mastrobuono-Battisti \& Perets 2016).

\subsubsection{Linear regression analysis}

By considering a linear combination of the logarithm of the cluster masses $\left(M_{i}\right)$, concentrations $\left(c_{i}\right)$, and rotational velocities $\left(v_{A, i}\right)$,

$F_{2}=\alpha_{0}+\alpha_{1} \log \left(M_{i}\right)+\alpha_{2} c_{i}+\alpha_{3} v_{A, i}$,

we performed a linear regression analysis that identified the mass as the most significant parameter determining $F_{2}\left(\alpha_{1}=5.6, p_{\text {value }}=\right.$ $\left.2.1 \times 10^{-6}\right)$. The concentration is more significant $\left(\alpha_{3}=1.3, p_{\text {value }}=\right.$ $0.20)$ than the rotational velocity $\left(\alpha_{3}=0.11, p_{\text {value }}=0.91\right)$. As only the $p_{\text {value }}$ for the mass is smaller than 0.05 and significantly smaller than the probabilities found for the other parameters, it appears that the cluster mass determines the $2 \mathrm{P}$ fraction, with little, if any, independent contribution from the other parameters, i.e. the observed correlations with the other parameters could arise solely from their correlation with the cluster mass.

All the correlations discussed above have high statistical significance at least for the entire sample of GCs and are suggestive of the fact that the $2 \mathrm{P}$ forms centrally concentrated inside the $1 \mathrm{P}$ cluster 
Table 2. Statistical correlation coefficients (PCC) and probabilities (PP) between the 3D rotational velocity amplitude, $v_{A}$, age, mass, and several GC internal cluster parameters. Parameters are reported for the entire sample of GCs and for clusters less massive (LM) and more massive (HM) than $10^{5.5} \mathrm{M}_{\odot}$. $\mathrm{P}$ stands for Pearson, $\mathrm{S}$ for Spearman, and $\mathrm{K}$ for Kendall.

\begin{tabular}{|c|c|c|c|c|c|c|c|c|}
\hline $\operatorname{Par}_{1}$ & $\mathrm{Par}_{2}$ & Stat & PCC & PP & $\mathrm{PCC}_{\mathrm{LM}}$ & $\mathrm{PP}_{\mathrm{LM}}$ & $\mathrm{PCC}_{\mathrm{HM}}$ & $\mathrm{PP}_{\mathrm{HM}}$ \\
\hline \multirow[t]{3}{*}{$\left|v_{A}\right|$} & \multirow[t]{3}{*}{$M$} & $\mathrm{P}$ & 0.47 & $1.5 \times 10^{-4}$ & 0.068 & 0.74 & 0.41 & 0.015 \\
\hline & & S & 0.51 & $3.1 \times 10^{-5}$ & -0.088 & 0.67 & 0.48 & 0.0032 \\
\hline & & K & 0.34 & $8.2 \times 10^{-5}$ & -0.074 & 0.61 & 0.32 & 0.0076 \\
\hline \multirow[t]{3}{*}{$\left|v_{A}\right|$} & \multirow[t]{3}{*}{$c$} & $\mathrm{P}$ & 0.43 & 0.0011 & 0.29 & 0.18 & 0.32 & 0.069 \\
\hline & & S & 0.39 & 0.0026 & 0.094 & 0.67 & 0.20 & 0.28 \\
\hline & & K & 0.27 & 0.0034 & 0.044 & 0.79 & 0.13 & 0.30 \\
\hline \multirow[t]{3}{*}{$\left|v_{A}\right|$} & \multirow[t]{3}{*}{ Age } & $\mathrm{P}$ & 0.083 & 0.61 & 0.16 & 0.95 & 0.18 & 0.43 \\
\hline & & S & 0.13 & 0.41 & 0.071 & 0.77 & 0.23 & 0.31 \\
\hline & & K & 0.077 & 0.50 & 0.085 & 0.65 & 0.13 & 0.44 \\
\hline \multirow[t]{3}{*}{$\left|v_{A}\right|$} & \multirow[t]{3}{*}{ Age $/ t_{\mathrm{rh}}$} & $\mathrm{P}$ & -0.33 & 0.038 & -0.21 & 0.38 & -0.42 & 0.055 \\
\hline & & S & -0.30 & 0.060 & -0.072 & 0.77 & -0.44 & 0.042 \\
\hline & & $\mathrm{K}$ & -0.22 & 0.047 & -0.041 & 0.84 & -0.33 & 0.034 \\
\hline \multirow[t]{3}{*}{$\left|v_{A}\right|$} & \multirow[t]{3}{*}{$\varepsilon$} & $\mathrm{P}$ & 0.21 & 0.11 & 0.079 & 0.73 & 0.26 & 0.13 \\
\hline & & S & 0.26 & 0.048 & 0.26 & 0.24 & 0.30 & 0.083 \\
\hline & & $\mathrm{K}$ & 0.19 & 0.045 & 0.20 & 0.21 & 0.22 & 0.074 \\
\hline \multirow[t]{3}{*}{$M$} & \multirow[t]{3}{*}{$V / \sigma$} & $\mathrm{P}$ & 0.16 & 0.28 & 0.34 & 0.13 & -0.065 & 0.75 \\
\hline & & S & 0.34 & 0.019 & 0.29 & 0.20 & 0.079 & 0.70 \\
\hline & & K & 0.21 & 0.037 & 0.18 & 0.26 & 0.038 & 0.80 \\
\hline \multirow[t]{3}{*}{$M$} & \multirow[t]{3}{*}{$c$} & $\mathrm{P}$ & 0.30 & 0.0021 & 0.25 & 0.049 & 0.10 & 0.53 \\
\hline & & S & 0.47 & $5.3 \times 10^{-7}$ & 0.37 & 0.0028 & 0.23 & 0.16 \\
\hline & & $\mathrm{K}$ & 0.34 & $3.6 \times 10^{-7}$ & 0.26 & 0.0027 & 0.16 & 0.14 \\
\hline \multirow[t]{3}{*}{$M$} & \multirow[t]{3}{*}{ Age } & $\mathrm{P}$ & 0.010 & 0.94 & 0.34 & 0.059 & -0.031 & 0.88 \\
\hline & & S & 0.030 & 0.83 & 0.23 & 0.22 & 0.89 & 0.72 \\
\hline & & $\mathrm{K}$ & $8.8 \times 10^{-3}$ & 0.93 & 0.16 & 0.23 & 0.00 & 1.00 \\
\hline \multirow[t]{3}{*}{$M$} & \multirow[t]{3}{*}{ Age $/ t_{\mathrm{rh}}$} & $\mathrm{P}$ & -0.32 & 0.019 & -0.15 & 0.44 & -0.65 & $8.3 \times 10^{-4}$ \\
\hline & & S & -0.27 & 0.047 & 0.083 & 0.66 & -0.75 & $3.5 \times 10^{-5}$ \\
\hline & & K & -0.20 & 0.036 & 0.058 & 0.66 & -0.56 & $2.4 \times 10^{-4}$ \\
\hline \multirow[t]{3}{*}{$M$} & \multirow[t]{3}{*}{$\varepsilon$} & $\mathrm{P}$ & 0.17 & 0.096 & 0.065 & 0.64 & 0.28 & 0.064 \\
\hline & & S & 0.17 & 0.10 & 0.15 & 0.29 & 0.16 & 0.31 \\
\hline & & $\mathrm{K}$ & 0.13 & 0.076 & 0.11 & 0.27 & 0.13 & 0.24 \\
\hline
\end{tabular}

and the fraction of $2 \mathrm{P}$ stars are mainly correlated to the mass. There is also a correlation with the amplitude of tidal stripping experienced by the clusters during their evolution, through their concentration parameter. The stronger the tidal effects, the more $1 \mathrm{P}$ stars - which are less concentrated - are stripped away increasing the relative $2 \mathrm{P}$ fraction, mostly distributed within the central region of the cluster, in the remnant stellar population. In such a case, the relaxation time has only a small influence on this process, at least for the less massive clusters (see Section 3.2). For shorter relaxation times we might expect a stronger mixing between the populations and therefore a somewhat lower increase of $F_{2}$ due to stripping (see Section 3.2).

\subsection{Multiple populations minor correlations with cluster properties}

Fig. 2 shows $F_{2}$ plotted against additional cluster parameters. The statistical significance of the possible correlations existing between these quantities is listed in Table 2.

The $F_{2}$ versus $|V / \sigma|^{1}$ plot shows several sequences and a positive trend. However, our tests do not detect a statistically significant correlation between these quantities, except for a slight trend that is, however, found only when considering the entire GC sample. We

\footnotetext{
${ }^{1}$ The $|V / \sigma|$ parameter is a measure of the ordered motion with respect to the velocity dispersion. As such, it gives an estimate of the rotation strength independently of the cluster mass.
}

note that Bellazzini et al. (2012) found a slight trend between the extent of the $\mathrm{Na}-\mathrm{O}$ anticorrelation and $V / \sigma$.

Although by eye there seem to be a positive trend of $F_{2}$ with the ellipticity of the clusters $(\varepsilon)$, this qualitative observation is statistically confirmed only for the high-mass clusters. The lack of correlation for the entire sample of GCs and for low-mass clusters might be due to the large spread of $F_{2}$ among low-mass GCs with similar ellipticities and to the existence of several outliers.

While the age of the clusters is not correlated to the $2 \mathrm{P}$ fraction, $F_{2}$ shows an anticorrelation with the dynamical age of the clusters (i.e. the age of the cluster in terms of half-mass relaxation times, age $/ t_{\mathrm{rh}}$ ) when considering the entire sample of clusters and only in the case of the Pearson statistical test. In Appendix C, we discuss the implication of using the different age compilations available in literature (Dotter et al. 2010; Forbes \& Bridges 2010; Gratton et al. 2010)

$F_{2}$ and the half-mass relaxation time $\left(t_{\mathrm{rh}}\right)$ of the high-mass clusters are correlated. The detected correlation is stronger when performing a Pearson and Spearman statistical test.

We also checked for the existence of a possible correlation between the number of orbits, i.e. of pericentre passages, travelled by cluster and $F_{2}$. The number of orbits has been evaluated as the ratio between the age and the radial period, $T_{r}$, of the cluster taken from Model 1 in Gaia Collaboration et al. (2018). Interestingly, these two quantities are anticorrelated when taking into account the lower mass clusters. The same anticorrelation is found when using the ages provided by 

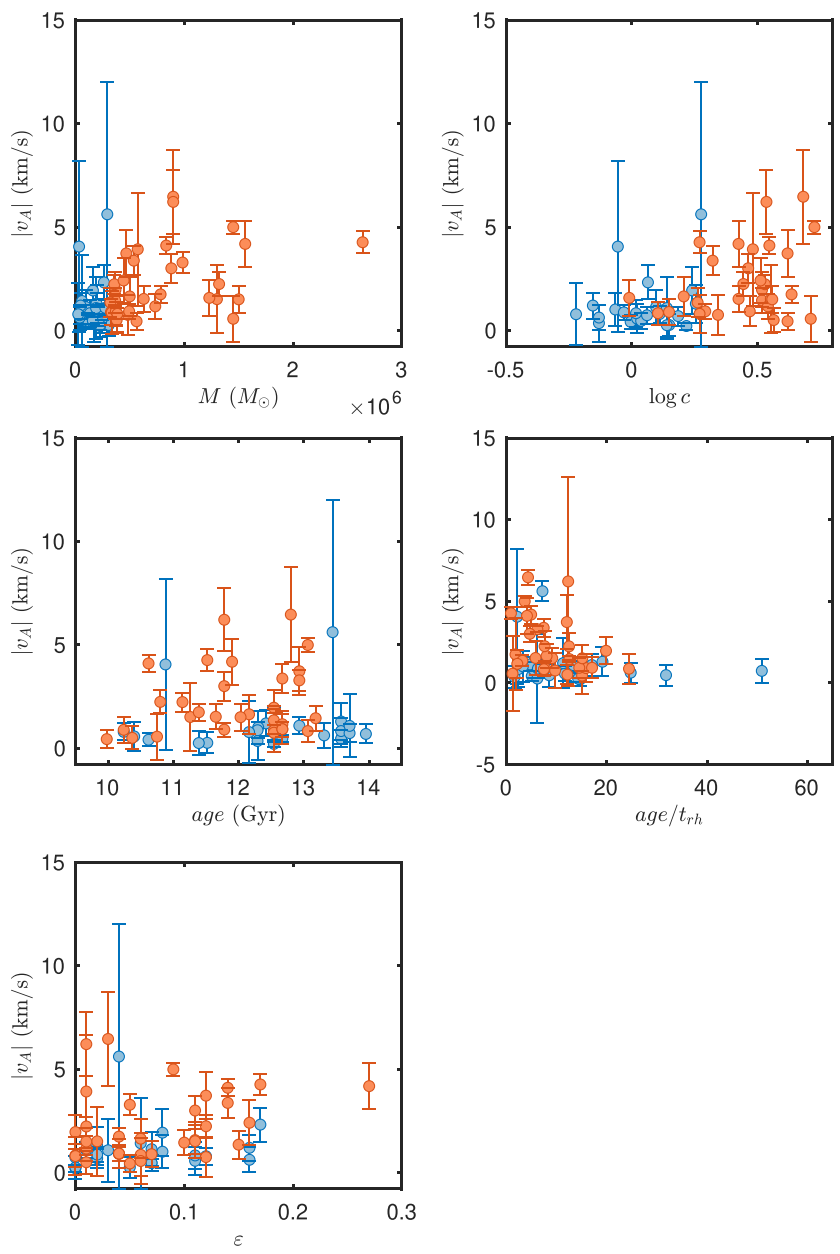

Figure 3. The $3 \mathrm{D}$ amplitude of the rotational velocity for the available cluster sample, $\left|v_{A}\right|$, is plotted against the mass of the clusters (top left-hand panel), the logarithm of the concentration parameter (top right-hand panel), the cluster age (middle left-hand panel), the dynamical age of the clusters (middle right-hand panel), and the cluster ellipticity (bottom left-hand panel). Orange symbols are clusters with masses larger than $10^{5.5} \mathrm{M}_{\odot}$, and light blue symbols are clusters with masses smaller than the same threshold.

Gratton et al. (2010) but not when using the ages from Dotter et al. (2010) and Forbes \& Bridges (2010).

\subsection{Correlations between cluster rotations, ages, and masses}

We checked for the existence of mutual correlations between the internal cluster parameters focusing on the rotation, concentration, mass, age related parameters, and ellipticity (see Figs 3 and 4 and Table 2).

The $3 \mathrm{D}$ rotational amplitude $\left(v_{A}\right)$ is strongly correlated with the cluster mass. The concentration and $v_{A}$ are correlated only when considering the whole GC sample and in the case of the Kendal statistical test performed for low-mass clusters.

The $3 \mathrm{D}$ rotational amplitude is not correlated with the age of the clusters. Interestingly, $v_{A}$ is anticorrelated with the dynamical age of the clusters when considering the entire sample of GCs or highmass clusters only. Since clusters with larger relaxation times have typically smaller dynamical ages, our result is in agreement with the positive correlation found between the rotational velocities and the cluster relaxation times by Kamann et al. (2018). The amplitude of the rotational velocity is correlated with the ellipticity of the clusters
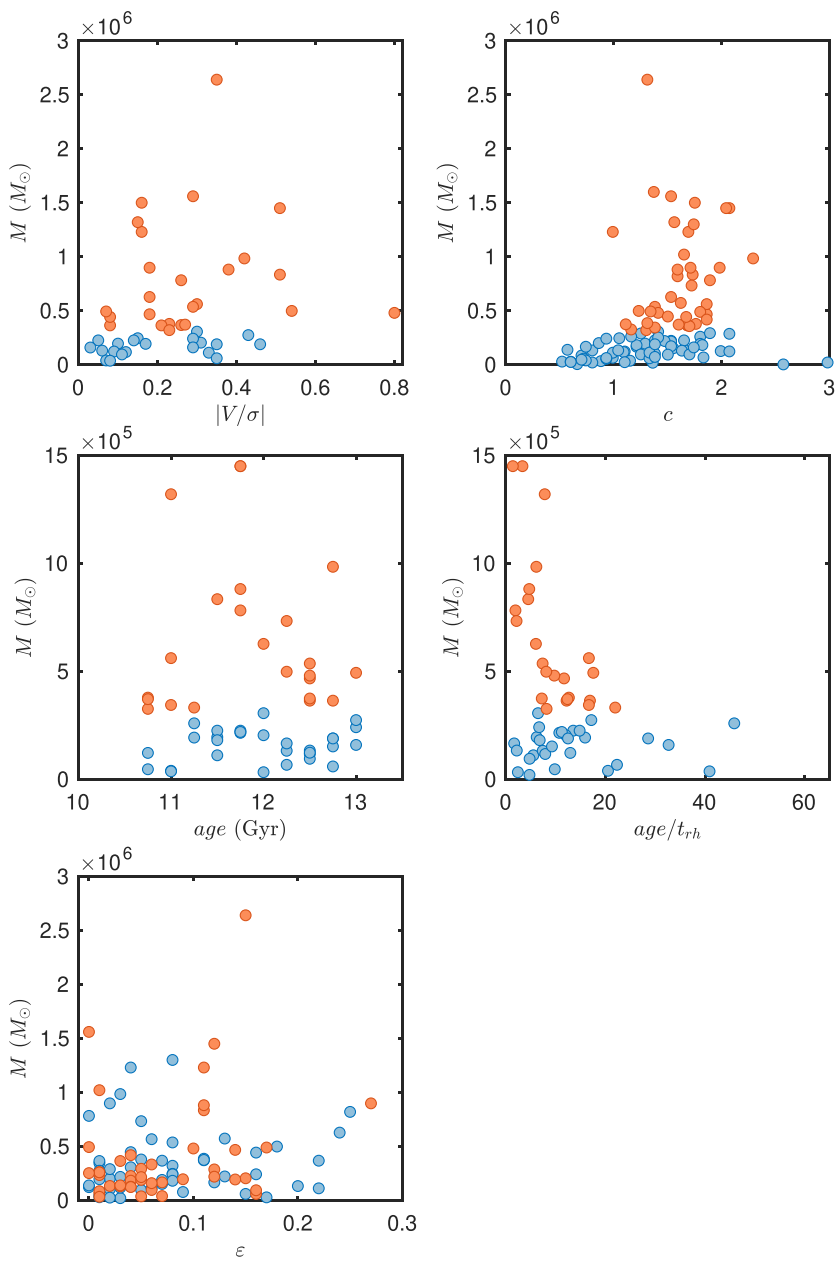

Figure 4. The GC masses are plotted against the clusters $|V / \sigma|$ parameter (top left-hand panel), the concentration (top right-hand panel), the age (middle lefthand panel), the dynamical age (middle right-hand panel), and the ellipticity (bottom left-hand panel) of the clusters. Orange symbols are clusters with masses larger than $10^{5.5} \mathrm{M}_{\odot}$, and light blue symbols are clusters with masses smaller than the same threshold. Errors for the masses are not available for the data set used.

$(\varepsilon)$ for the entire GC sample only when removing the outliers. We should, however, caution that here we are considering the projected ellipticities. Therefore, this result could be significantly affected by projection effects that may smear underlying correlations.

The $V / \sigma$ parameter, which measures the strength of the rotation of the system compared to its velocity dispersion, seems to be mildly correlated with the current cluster mass as also found by Bianchini et al. (2018) and Sollima et al. (2019). This is true only for the whole sample of GCs and after removing the effect of the outliers. The concentration and the mass are strongly correlated when considering the whole sample of GCs and low-mass GCs only.

The mass and the age are not correlated. However, the mass is strongly correlated with the dynamical age of high-mass clusters. Finally, the mass is not significantly correlated with the ellipticity of the clusters.

\section{DISCUSSION AND CONCLUSIONS}

GCs with ages larger than their relaxation times should still show signatures of their primordial structure, including those left by the $2 \mathrm{P}$ 


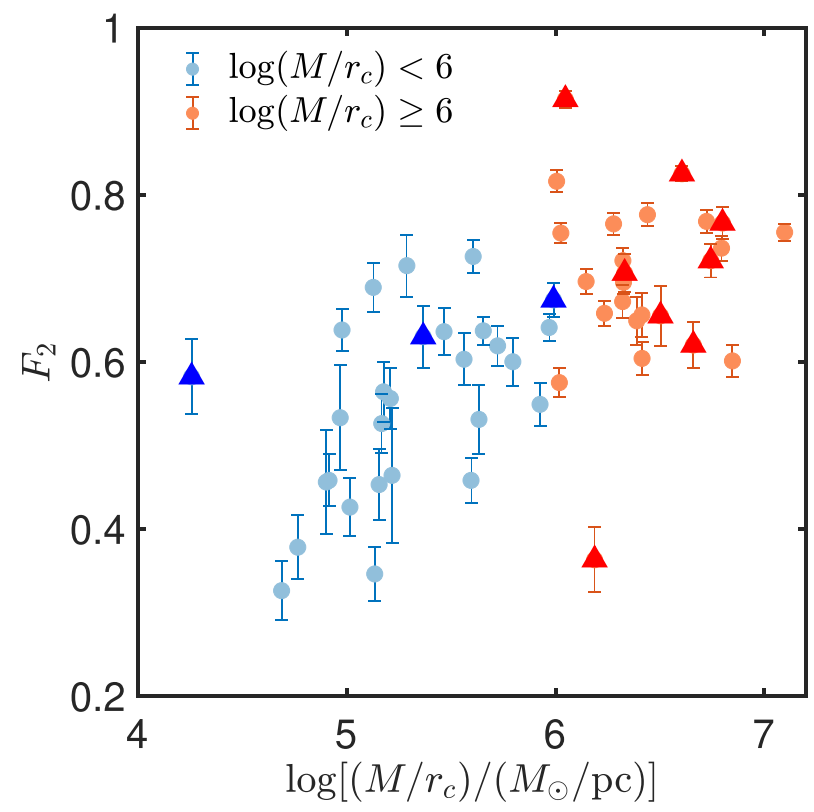

Figure 5. The second generation fraction, $F_{2}$, is plotted against an indicator of the escape velocity of the cluster, $\log \left(M / r_{\mathrm{c}}\right)$. Light blue symbols are clusters with $\log \left(M / r_{\mathrm{c}}\right)<6$, and orange symbols are clusters with $\log \left(M / r_{\mathrm{c}}\right) \geq 6$. Triangles are core-collapsed clusters and clusters suspected to be accreted nuclear star clusters (NSCs).

stars formation mechanism (see e.g. Mastrobuono-Battisti \& Perets 2013, 2016; Vesperini et al. 2013, 2021; Hénault-Brunet et al. 2015; Cordero et al. 2017; Tiongco et al. 2019).

By studying the relationships between global parameters [e.g. the horizontal branch (HB) morphology and structural or orbital parameters] and the abundance patterns of the different populations within each GC, Carretta et al. (2010) revised the GC formation mechanism dividing it into three phases: the formation of a precursor population is followed by the birth of the primordial population and then by the formation of the current GC, mainly from gas lost through the slow winds of a fraction of the primordial population. This scenario implies that the initial clusters were several times more massive than the current ones and that the halo of the Galaxy could contain a large fraction of former GC stars. This study relied on a set of homogeneous chemical abundances obtained for more than 1200 red giants in 19 clusters, as well as on additional data from literature and parameters coming from Harris (1996, 2010 edition). Through their approach, Carretta et al. (2010), found that the extent of the $\mathrm{Na}-\mathrm{O}$ anticorrelation, calculated as the interquartile range (IQR[O/Na]), is mainly linked to the mass, age, and metallicity of the clusters. Carretta (2019) used 22 GCs observed with the Fibre Large Array Multi Element Spectrograph (FLAMES) to calibrate the observed IQR[O/Na] values with respect to structural parameters and HB morphology of 120 Galactic GCs. In such a way, they found that the main parameters linked to the $2 \mathrm{P}$ phenomenon are the initial mass and the concentration of the clusters. Bellazzini et al. (2012) found a hint of a negative trend between IQR $[\mathrm{O} / \mathrm{Na}]$ and $V / \sigma$. However, they did not detect any correlation between the $2 \mathrm{P}$ and the rotational velocities of the observed clusters.

Milone et al. $(2017,2020)$ found a strong anticorrelation between the $1 \mathrm{P}$ stellar fraction and both the mass of the $2 \mathrm{P}$ component and the cluster initial mass. They found as well a milder correlation between the current cluster mass and that of the $2 \mathrm{P}$ (see also Dondoglio et al. 2021). The luminosity and perigalactic radii of the clusters also might play a role being correlated with the $1 \mathrm{P}$ fraction and helium variations, respectively. Dondoglio et al. (2021) recently found that two clusters in the Magellanic Clouds show a smaller fraction of $2 \mathrm{P}$ stars, suggesting a link between the multiple population phenomenon and environmental effects as the strength of the tidal forces exerted by host galaxy on the clusters.

In this work, we collected GC internal parameters from literature to search for possible correlations with the fraction of second generation stars observed in each cluster. For the first time, we carry out a homogeneous statistical search to study all of these various parameters together and on the same extended sample.

We confirm the existence of a strong correlation between the second generation number fraction and the cluster mass. This tight relationship becomes bimodal and more scattered at lower cluster masses. This clear effect could be linked to the cluster conditions at the epoch of $2 \mathrm{P}$ formation and to their subsequent evolution. A positive trend of $F_{2}$ with the present-day and initial mass of the clusters has been already observed by Milone et al. (2017, 2020), Lagioia et al. (2019), Carretta (2019), and Gratton et al. (2019) and it is expected if $2 \mathrm{P}$ stars form from material lost from 1P stars.

We observe a correlation between the $2 \mathrm{P}$ stellar fraction and the rotational velocity of the observed clusters. Such correlation is expected if $2 \mathrm{P}$ stars form centrally concentrated from gas lost by $1 \mathrm{P}$ stars collected in a rotating disc at the cluster centre ${ }^{2}$ (Hénault-Brunet et al. 2015; Mastrobuono-Battisti \& Perets 2016). To verify a direct connection with the formation process it is necessary to check the rotation of the $1 \mathrm{P}$ and $2 \mathrm{P}$ separately, to identify a differential rotation between the two populations. This has been done for few clusters, however, since the analysed clusters show significantly different behaviours, no firm conclusion has been yet achieved (Milone et al. 2018; Cordoni et al. 2020a,b; Szigeti et al. 2021). Additionally, the strong correlation existing between cluster mass and rotation makes it difficult to disentangle between the relevance of these two properties with respect to the $2 \mathrm{P}$ origin.

A larger $F_{2}$ is observed for clusters with growing concentrations. In a scenario where $2 \mathrm{P}$ form at the cluster centre, a larger concentration corresponds to a more centrally bound second stellar population and to a stronger tidal stripping affecting the more radially extended $1 \mathrm{P}$ component. Therefore, a more significant $1 \mathrm{P}$ mass loss is expected compared with less concentrated clusters. As previously also pointed out by Richer et al. (2013), Bellini et al. (2015), Cordero et al. (2017), Kamann et al. (2020), and Dalessandro et al. (2019), the correlation existing between $F_{2}$ and the concentration parameter supports the idea that $2 \mathrm{P}$ stars are born concentrated at the centre of the cluster, while the initial radial distribution of $1 \mathrm{P}$ stars is more extended. The more the cluster is concentrated, the more $1 \mathrm{P}$ stars will be stripped away. This possibility has been mostly dynamically and hydrodynamically tested in the case of the AGB scenario (see e.g. D'Ercole et al. 2008; Bekki 2010, 2011; MastrobuonoBattisti \& Perets 2013, 2016; Hénault-Brunet et al. 2015; Calura et al. 2019; Tiongco et al. 2019; Vesperini et al. 2021). However, such relationship might be expected in any self-enrichment scenario.

Our regression analysis points at the mass as the main parameter that determines the fraction of $2 \mathrm{P}$ stars. Other correlations observed

\footnotetext{
${ }^{2}$ Qualitatively, we expect that when a larger quantity of gas (bringing in the orbital angular momentum inherited from the donor) collects at the centre of the cluster, a larger net angular momentum should be gained by the forming $2 \mathrm{P}$ stars. A growing $F_{2}$ should, therefore, correspond to a growing rotational velocity.
} 
Table 3. Statistical correlation coefficients (PCC) and probabilities (PP) between $F_{2}$ and an escape velocity indicator calculated as the mass of the cluster over its core radius $\left(M / r_{\mathrm{c}}\right)$. Parameters are reported for the entire sample of GCs, for clusters with $M / r_{\mathrm{c}}$ smaller (LV) and higher (HV) than $10^{6} \mathrm{M}_{\odot} \mathrm{pc}^{-1}$. P stands for Pearson, $\mathrm{S}$ for Spearman, and $\mathrm{K}$ for Kendall. The first three rows are for the entire sample of GCs, the last three rows are calculated excluding core-collapsed clusters and clusters suspected to be accreted nuclear star clusters (NSCs).

\begin{tabular}{ccccccccc}
\hline $\mathrm{Par}_{1}$ & $\mathrm{Par}_{2}$ & $\mathrm{Stat}$ & $\mathrm{PCC}$ & $\mathrm{PP}$ & $\mathrm{PCC}_{\mathrm{LV}}$ & $\mathrm{PP}_{\mathrm{LV}}$ & $\mathrm{PCC}_{\mathrm{HV}}$ & $\mathrm{PP}_{\mathrm{HV}}$ \\
\hline$F_{2}$ & $\log \left(M / r_{\mathrm{c}}\right)$ & $\mathrm{P}$ & 0.47 & $1.5 \times 10^{-4}$ & 0.41 & 0.0019 & 0.13 & 0.55 \\
& & $\mathrm{~S}$ & 0.67 & $2.9 \times 10^{-8}$ & 0.49 & 0.0074 & 0.24 & 0.24 \\
& & $\mathrm{~K}$ & 0.48 & $3.1 \times 10^{-7}$ & 0.26 & 0.0062 & 0.15 & 0.30 \\
$F_{2}$ & $\log \left(M / r_{\mathrm{c}}\right)$ & $\mathrm{P}$ & 0.45 & 0.0027 & 0.43 & 0.034 & 0.11 & 0.67 \\
& & $\mathrm{~S}$ & 0.71 & $1.2 \times 10^{-7}$ & 0.56 & 0.0039 & -0.0098 & 0.97 \\
& & $\mathrm{~K}$ & 0.53 & $1.1 \times 10^{-6}$ & 0.41 & 0.0044 & -0.059 & 0.78 \\
\hline
\end{tabular}

with $F_{2}$ appear to be mediated by the mass e.g. with the rotational velocity or the concentration.

While Lagioia et al. (2019) find a marginal correlation between the presence of $2 \mathrm{P}$ stars and ages of Galactic and Magellanic Clouds clusters, Martocchia et al. $(2018,2019)$ results suggest that the age has a crucial role in shaping the characteristics of multiple populations. Older clusters, in fact, show larger nitrogen spreads compared to younger ones. However, with the available data we do not detect any correlation between the cluster age and $F_{2}$.

In principle, clusters that experience a larger number of pericentre passages might be somewhat more stripped, though such an effect could be secondary compared with other parameters. The number of pericentre passages is measured by the age of the clusters divided by their radial orbital periods around the Galaxy, $T_{r}$. We find that lowmass clusters show a correlation between $F_{2}$ and age/ $T_{r}$. In general, low-mass clusters are more affected by tidal stripping than high-mass clusters possibly explaining why we observe the correlation only for clusters less massive than $10^{5.5} \mathrm{M}_{\odot}$. The effect of the tidal stripping on the fraction of $2 \mathrm{P}$ stars is also confirmed by the fact that clusters with large perigalactic distances host, on average, a smaller $F_{2}$ (Zennaro et al. 2019). In contrast, D'Ercole et al. (2008) and, more recently, Vesperini et al. (2021) using $N$-body simulations of the cluster evolution, suggest that stellar evolution and primordial mass segregation could be the major causes of the preferential loss of 1P stars.

$F_{2}$ is mildly anticorrelated with the dynamical age (measured in relaxation times), especially when applying a Pearson statistical test to the entire sample of clusters. This can be understood if $1 \mathrm{P}$ and $2 \mathrm{P}$ stars in dynamically older clusters are more mixed than in younger clusters. In dynamically older GCs, then, tidal stripping can become more efficient in removing $2 \mathrm{P}$ stars that, in such clusters, are more abundant in the cluster outskirts.

We also verified the existence of a relation between the $A^{+}$ parameter provided by Dalessandro et al. (2019), which is a measure of the $2 \mathrm{P}$ concentration with respect to the $1 \mathrm{P}$, and $F_{2}$. We found that these two quantities are indeed anticorrelated, meaning that more concentrated $2 \mathrm{P}$ (i.e. less mixed populations or dynamically younger clusters) show lower $F_{2} . F_{2}$ and the half-mass relaxation time $\left(t_{\mathrm{rh}}\right)$ are correlated for high-mass clusters. For these clusters, relaxation effects might have been more important than the tidal interaction with the Galaxy.

All the detected (anti)correlations appear to be bimodal, with different behaviours for clusters less massive or more massive than $10^{5.5} \mathrm{M}_{\odot}$. These include the mass correlation, which is stronger for more massive clusters. The mass cut-off could be linked to the formation mechanism, which seems to be more efficient for more massive clusters, and also to the following evolution that affects differently clusters roughly below and above the mass cut-off. Metal- poor clusters $([\mathrm{Fe} / \mathrm{H}]<-1)$, i.e. possibly GCs accreted during mergers, show slightly stronger correlations with the mass, concentration, and rotational velocity than metal-rich clusters $([\mathrm{Fe} / \mathrm{H}] \geq-1)$. The remaining correlations do not show any trend with the metallicity (see Appendix A).

As a final test, we checked for the existence of a possible link between $F_{2}$ and the escape velocity of the clusters, estimated through an indicator $\left(M / r_{\mathrm{c}}\right.$, where $M$ is the mass of the cluster and $r_{\mathrm{c}}$ is its core radius).

As shown in Fig. 5 and Table 3, all the clusters taken as a single group show a strong correlation between the $2 \mathrm{P}$ fraction and the escape velocity of the clusters. A similar relationship has been also pointed out by Baumgardt \& Hilker (2018). Additionally, we observe that while clusters with $M / r_{\mathrm{c}} \geq 10^{6} \mathrm{M}_{\odot} \mathrm{pc}^{-1}$ do not show any correlation between these quantities, clusters with low escape velocity $\left(M / r_{\mathrm{c}}<10^{6} \mathrm{M}_{\odot} \mathrm{pc}^{-1}\right)$ display a correlation between $F_{2}$ and $\log \left(M / r_{\mathrm{c}}\right)$. This correlation that, for the high escape velocity clusters, is as strong as the one observed between $F_{2}$ and the cluster mass becomes slightly stronger when excluding core-collapsed clusters (Harris 1996) and GCs suspected to be nuclear star clusters (NSCs; Pfeffer et al. 2021).

In self-enrichment scenarios, the fraction of retained gas, and therefore $F_{2}$, should increase with the escape velocity from the cluster, i.e. with the depth of the central potential well. However, sufficiently massive and centrally concentrated clusters have escape velocities high enough as to potentially retain all the gas lost through stellar winds from evolved stars, leading to $2 \mathrm{P}$ fractions showing only small fluctuations around the mean value.

In conclusion, higher escape velocities, as well as higher masses, seem to correspond to a larger fraction of retained gas that, in turn, gives rise to larger fractions of $2 \mathrm{P}$ stars. The gas retained in the cluster core forms $2 \mathrm{P}$ stars, while $1 \mathrm{P}$ stars are more extended and more easily lost by the cluster in the interaction with the Galactic tidal field.

\section{ACKNOWLEDGEMENTS}

The authors thank the referee for the helpful and careful comments that improved this work and A. F. Marino and A. Milone for the useful discussion and comments. AM-B is supported by the Swedish Research Council (grant 2017-04217).

\section{DATA AVAILABILITY}

The data underlying this paper are available in the paper and in the cited literature. 


\section{REFERENCES}

Bastian N., Lardo C., 2018, ARA\&A, 56, 83

Bastian N., Lamers H. J. G. L. M., de Mink S. E., Longmore S. N., Goodwin S. P., Gieles M., 2013, MNRAS, 436, 2398

Baumgardt H., Hilker M., 2018, MNRAS, 478, 1520

Bekki K., 2010, ApJ, 724, L99

Bekki K., 2011, MNRAS, 412, 2241

Bellazzini M., Bragaglia A., Carretta E., Gratton R. G., Lucatello S., Catanzaro G., Leone F., 2012, A\&A, 538, A18

Bellini A. et al., 2015, ApJ, 810, L13

Bellini A. et al., 2018, ApJ, 853, 86

Bianchini P., van der Marel R. P., del Pino A., Watkins L. L., Bellini A., Fardal M. A., Libralato M., Sills A., 2018, MNRAS, 481, 2125

Breen P. G., 2018, MNRAS, 481, L110

Calura F., D’Ercole A., Vesperini E., Vanzella E., Sollima A., 2019, MNRAS, 489,3269

Carretta E., 2019, A\&A, 624, A24

Carretta E. et al., 2009, A\&A, 505, 117

Carretta E., Bragaglia A., Gratton R. G., Recio-Blanco A., Lucatello S., D’Orazi V., Cassisi S., 2010, A\&A, 516, A55

Carretta E. et al., 2014, A\&A, 564, A60

Cordero M. J., Pilachowski C. A., Johnson C. I., McDonald I., Zijlstra A. A., Simmerer J., 2014, ApJ, 780, 94

Cordero M. J., Hénault-Brunet V., Pilachowski C. A., Balbinot E., Johnson C. I., Varri A. L., 2017, MNRAS, 465, 3515

Cordoni G., Milone A. P., Mastrobuono-Battisti A., Marino A. F., Lagioia E. P., Tailo M., Baumgardt H., Hilker M., 2020a, ApJ, 889, 18

Cordoni G. et al., 2020b, ApJ, 898, 147

Cordoni G., Milone A. P., Mastrobuono-Battisti A., Marino A. F., Lagioia E. P., Tailo M., 2020c, in Bragaglia A., Davies M., Sills A., Vesperini E., eds, Proc. IAU Symp. Vol. 351, Star Clusters: From the Milky Way to the Early Universe. Cambridge Univ. Press, Cambridge, p. 281

Dalessandro E. et al., 2018, ApJ, 864, 33

Dalessandro E. et al., 2019, ApJ, 884, L24

Decressin T., Meynet G., Charbonnel C., Prantzos N., Ekström S., 2007, A\&A, 464, 1029

de Marchi G., Pulone L., 2007, A\&A, 467, 107

D’Ercole A., Vesperini E., D’Antona F., McMillan S. L. W., Recchi S., 2008, MNRAS, 391, 825

Dondoglio E., Milone A. P., Lagioia E. P., Marino A. F., Tailo M., Cordoni G., Jang S., Carlos M., 2021, ApJ, 906, 76

Dotter A. et al., 2010, ApJ, 708, 698

Forbes D. A., Bridges T., 2010, MNRAS, 404, 1203

Gaia Collaboration et al., 2018, A\&A, 616, A12

Gieles M. et al., 2018, MNRAS, 478, 2461

Gnedin O. Y., Ostriker J. P., 1997, ApJ, 474, 223

Gratton R. G., Carretta E., Bragaglia A., Lucatello S., D’Orazi V., 2010, A\&A, 517, A81

Gratton R. G., Carretta E., Bragaglia A., 2012, A\&AR, 20, 50

Gratton R., Bragaglia A., Carretta E., D’Orazi V., Lucatello S., Sollima A., 2019, A\&AR, 27, 8

Harris W. E., 1996, AJ, 112, 1487

Hénault-Brunet V., Gieles M., Agertz O., Read J. I., 2015, MNRAS, 450, 1164

Kamann S. et al., 2018, MNRAS, 473, 5591

Kamann S. et al., 2020, MNRAS, 492, 966

Lagioia E. P., Milone A. P., Marino A. F., Cordoni G., Tailo M., 2019, AJ, 158,202

Lee J.-W., 2017, ApJ, 844, 77

Lee J.-W., 2018, ApJS, 238, 24

Martocchia S. et al., 2018, MNRAS, 473, 2688

Martocchia S. et al., 2019, MNRAS, 487, 5324

Mastrobuono-Battisti A., Perets H. B., 2013, ApJ, 779, 85

Mastrobuono-Battisti A., Perets H. B., 2016, ApJ, 823, 61

Milone A. P. et al., 2012a, A\&A, 540, A16

Milone A. P., Marino A. F., Piotto G., Bedin L. R., Anderson J., Aparicio A., Cassisi S., Rich R. M., 2012b, ApJ, 745, 27
Milone A. P. et al., 2016, MNRAS, 455, 3009

Milone A. P. et al., 2017, MNRAS, 464, 3636

Milone A. P., Marino A. F., Mastrobuono-Battisti A., Lagioia E. P., 2018, MNRAS, 479, 5005

Milone A. P. et al., 2020, MNRAS, 491, 515

Norris J. E., Da Costa G. S., 1995, ApJ, 447, 680

Norris J., Freeman K. C., 1979, ApJ, 230, L179

Paust N. E. Q. et al., 2009, AJ, 137, 246

Pfeffer J., Lardo C., Bastian N., Saracino S., Kamann S., 2021, MNRAS, 500,2514

Piatti A. E., 2020, A\&A, 643, A77

Piotto G. et al., 2007, ApJ, 661, L53

Renzini A. et al., 2015, MNRAS, 454, 4197

Richer H. B., Heyl J., Anderson J., Kalirai J. S., Shara M. M., Dotter A., Fahlman G. G., Rich R. M., 2013, ApJ, 771, L15

Sollima A., Baumgardt H., Hilker M., 2019, MNRAS, 485, 1460

Szigeti L., Mészáros S., Szabó G. M., Fernández-Trincado J. G., Lane R. R., Cohen R. E., 2021, MNRAS, 504, 1144

Tiongco M. A., Vesperini E., Varri A. L., 2019, MNRAS, 487, 5535

VandenBerg D. A., Brogaard K., Leaman R., Casagrande L., 2013, ApJ, 775, 134

Ventura P., D’Antona F., Mazzitelli I., Gratton R., 2001, ApJ, 550, L65

Vesperini E., McMillan S. L. W., D'Antona F., D'Ercole A., 2013, MNRAS, 429,1913

Vesperini E., Hong J., Giersz M., Hypki A., 2021, MNRAS, 502, 4290

Wang L., Kroupa P., Takahashi K., Jerabkova T., 2020, MNRAS, 491, 440

Zennaro M., Milone A. P., Marino A. F., Cordoni G., Lagioia E. P., Tailo M., 2019, MNRAS, 487, 3239

\section{APPENDIX A: CORRELATIONS AND CLUSTER METALLICITIES}

We tested the same correlations studied in Section 3 dividing the clusters into two groups depending on their metallicities instead of their masses. Low-metallicity clusters have $[\mathrm{Fe} / \mathrm{H}]<-1$, and highmetallicity clusters have $[\mathrm{Fe} / \mathrm{H}] \geq-1$.

The results of this analysis are presented in Figs A1 and A2. and in Tables A1 and A2. The differences in the statistical parameters obtained for the entire sample of GCs when using either the masses or the metallicities are due to the fact that these quantities are not available for the same clusters. We do not observe any strong trend of the correlations with the metallicity.

Most of the correlations remain valid (or absent) for both groups when splitting the GCs according to their metallicities. The amplitude of the rotational velocity is correlated to $F_{2}$ only when considering the entire GC sample and for metal-poor clusters. This might be linked to the conditions at formation for in situ clusters (see e.g. Piatti 2020) or, more likely, simply be caused by the low number of clusters belonging to the metal-rich subgroup. The dynamical age of the clusters seems correlated to $F_{2}$ only in the case of the Pearson statistical test and when considering all the clusters and metal-poor clusters only.

We detect a slight anticorrelation between $F_{2}$ and the number of orbits travelled by the clusters, age $/ T_{r}$ : the Kendal $p_{\text {value }}$ is just above 0.05 for the metal-rich clusters.

Interestingly, metal-rich clusters sit at the edge of the metal-poor cluster population in the $F_{2}$-dynamical age plane.

The $V / \sigma$ parameter is not correlated to $F_{2}$ when separating them in two metallicity subgroups.

The concentration and the mass are strongly correlated for the entire sample of GCs and they are less strongly correlated to the $2 \mathrm{P}$ fraction for the metal-rich clusters than for the metal-poor GCs. The mass and the concentration are mutually correlated for the entire 
Table A1. Statistical correlation coefficients (PCC) and probabilities (PP) between $F_{2}$ and several GC internal parameters. Parameters are reported for the entire sample of GCs, for metal-poor (MP) and metal-rich (MR) clusters. P stands for Pearson, S for Spearman, and $\mathrm{K}$ for Kendall.

\begin{tabular}{|c|c|c|c|c|c|c|c|c|}
\hline $\mathrm{Par}_{1}$ & $\mathrm{Par}_{2}$ & Stat & PCC & PP & $\mathrm{PCC}_{\mathrm{MP}}$ & $\mathrm{PP}_{\mathrm{MP}}$ & $\mathrm{PCC}_{\mathrm{MR}}$ & $\mathrm{PP}_{\mathrm{MR}}$ \\
\hline \multirow[t]{3}{*}{$\overline{F_{2}}$} & $M$ & $P$ & 0.76 & $3.2 \times 10^{-11}$ & 0.70 & $1.0 \times 10^{-7}$ & 0.86 & 0.0016 \\
\hline & & $S$ & 0.77 & $1.5 \times 10^{-11}$ & 0.70 & $1.1 \times 10^{-7}$ & 0.78 & 0.012 \\
\hline & & $\mathrm{K}$ & 0.58 & $4.9 \times 10^{-10}$ & 0.53 & $4.0 \times 10^{-7}$ & 0.64 & 0.0091 \\
\hline \multirow[t]{3}{*}{$F_{2}$} & $c$ & $\mathrm{P}$ & 0.50 & $2.9 \times 10^{-4}$ & 0.35 & 0.027 & 0.83 & 0.0059 \\
\hline & & $S$ & 0.55 & $4.5 \times 10^{-5}$ & 0.41 & 0.0089 & 0.77 & 0.021 \\
\hline & & $\mathrm{K}$ & 0.40 & $6.2 \times 10^{-5}$ & 0.30 & 0.0078 & 0.61 & 0.025 \\
\hline \multirow[t]{3}{*}{$F_{2}$} & $\left|v_{t}\right|$ & $\mathrm{P}$ & 0.46 & 0.0044 & 0.31 & 0.10 & 0.78 & 0.037 \\
\hline & & $\mathrm{S}$ & 0.54 & $8.7 \times 10^{-4}$ & 0.35 & 0.060 & 0.61 & 0.17 \\
\hline & & $\mathrm{K}$ & 0.38 & $8.0 \times 10^{-4}$ & 0.26 & 0.053 & 0.52 & 0.13 \\
\hline \multirow[t]{3}{*}{$F_{2}$} & $\left|v_{A}\right|$ & $P$ & 0.47 & 0.0016 & 0.45 & 0.0064 & 0.61 & 0.15 \\
\hline & & $\mathrm{S}$ & 0.43 & 0.0042 & 0.36 & 0.033 & 0.54 & 0.24 \\
\hline & & $\mathrm{K}$ & 0.30 & 0.0052 & 0.26 & 0.032 & 0.43 & 0.24 \\
\hline \multirow[t]{3}{*}{$F_{2}$} & $V / \sigma$ & $\mathrm{P}$ & 0.28 & 0.11 & 0.052 & 0.79 & 0.74 & 0.091 \\
\hline & & $\mathrm{S}$ & 0.33 & 0.056 & 0.099 & 0.61 & 0.60 & 0.24 \\
\hline & & $\mathrm{K}$ & 0.21 & 0.083 & 0.057 & 0.68 & 0.47 & 0.27 \\
\hline \multirow[t]{3}{*}{$F_{2}$} & $\varepsilon$ & $\mathrm{P}$ & 0.082 & 0.57 & 0.18 & 0.27 & -0.048 & 0.90 \\
\hline & & $\mathrm{S}$ & 0.079 & 0.58 & 0.14 & 0.38 & 0.055 & 0.88 \\
\hline & & $\mathrm{K}$ & 0.058 & 0.57 & 0.10 & 0.36 & 0.046 & 0.93 \\
\hline \multirow[t]{3}{*}{$F_{2}$} & Age & $\mathrm{P}$ & 0.0035 & 0.98 & -0.21 & 0.20 & 0.19 & 0.63 \\
\hline & & $\mathrm{S}$ & -0.050 & 0.74 & -0.23 & 0.14 & 0.53 & 0.15 \\
\hline & & $\mathrm{K}$ & -0.39 & 0.72 & -0.16 & 0.16 & 0.51 & 0.084 \\
\hline \multirow[t]{3}{*}{$F_{2}$} & $\mathrm{Age} / t_{\mathrm{rh}}$ & $\mathrm{P}$ & -0.31 & 0.031 & -0.35 & 0.029 & -0.064 & 0.87 \\
\hline & & S & -0.17 & 0.26 & -0.075 & 0.65 & 0.083 & 0.84 \\
\hline & & K & -0.11 & 0.27 & -0.048 & 0.67 & 0.17 & 0.61 \\
\hline \multirow[t]{3}{*}{$F_{2}$} & $\log \left(t_{\mathrm{rh}}\right)$ & $\mathrm{P}$ & 0.25 & 0.071 & 0.22 & 0.15 & 0.20 & 0.57 \\
\hline & & $\mathrm{S}$ & 0.080 & 0.61 & -0.018 & 0.97 & 0.14 & 0.31 \\
\hline & & $\mathrm{K}$ & 0.093 & 0.32 & 0.047 & 0.66 & -0.067 & 0.86 \\
\hline \multirow[t]{3}{*}{$F_{2}$} & $\mathrm{Age} / T_{r}$ & $\mathrm{P}$ & -0.025 & 0.88 & -0.031 & 0.87 & 0.61 & 0.20 \\
\hline & & $\mathrm{S}$ & -0.021 & 0.90 & -0.011 & 0.96 & 0.83 & 0.58 \\
\hline & & $\mathrm{K}$ & -0.015 & 0.91 & -0.011 & 0.95 & 0.73 & 0.056 \\
\hline
\end{tabular}

Table A2. Statistical correlation coefficients (PCC) and probabilities (PP) between the rotational velocity $v_{A}$, age, and several GC internal parameters. Parameters are reported for the entire sample of GCs, for metal-poor (MP) and metal-rich (MR) clusters. P stands for Pearson, S for Spearman, and K for Kendall.

\begin{tabular}{|c|c|c|c|c|c|c|c|c|}
\hline $\operatorname{Par}_{1}$ & $\mathrm{Par}_{2}$ & Stat & PCC & PP & $\mathrm{PCC}_{\mathrm{MP}}$ & $\mathrm{PP}_{\mathrm{MP}}$ & $\mathrm{PCC}_{\mathrm{MR}}$ & $\mathrm{PP}_{\mathrm{MR}}$ \\
\hline \multirow[t]{3}{*}{$\left|v_{A}\right|$} & $M$ & $\mathrm{P}$ & 0.47 & $1.5 \times 10^{-4}$ & 0.45 & 0.0014 & 0.53 & 0.050 \\
\hline & & $\mathrm{S}$ & 0.51 & $3.1 \times 10^{-5}$ & 0.51 & $2.4 \times 10^{-4}$ & 0.60 & 0.026 \\
\hline & & $\mathrm{K}$ & 0.35 & $8.2 \times 10^{-5}$ & 0.36 & $3.2 \times 10^{-4}$ & 0.41 & 0.047 \\
\hline \multirow[t]{3}{*}{$\left|v_{A}\right|$} & $c$ & $\mathrm{P}$ & 0.41 & 0.0017 & 0.39 & 0.0092 & 0.45 & 0.11 \\
\hline & & $\mathrm{S}$ & 0.40 & 0.0023 & 0.34 & 0.025 & 0.62 & 0.018 \\
\hline & & $\mathrm{K}$ & 0.27 & 0.0033 & 0.25 & 0.021 & 0.41 & 0.048 \\
\hline \multirow[t]{3}{*}{$\left|v_{A}\right|$} & Age & $\mathrm{P}$ & 0.049 & 0.73 & 0.11 & 0.49 & -0.092 & 0.80 \\
\hline & & $\mathrm{S}$ & 0.18 & 0.25 & -0.57 & 0.089 & 0.065 & 0.64 \\
\hline & & $\mathrm{K}$ & -0.055 & 0.61 & 0.054 & 0.66 & -0.32 & 0.30 \\
\hline \multirow{3}{*}{$\left|v_{A}\right|$} & Age $/ t_{\mathrm{rh}}$ & $\mathrm{P}$ & -0.28 & 0.040 & -0.23 & 0.13 & -0.46 & 0.19 \\
\hline & & $\mathrm{S}$ & -0.29 & 0.034 & -0.25 & 0.097 & -0.45 & 0.19 \\
\hline & & $\mathrm{K}$ & -0.20 & 0.028 & -0.18 & 0.086 & -0.42 & 0.11 \\
\hline \multirow[t]{3}{*}{$\left|v_{A}\right|$} & $\varepsilon$ & $\mathrm{P}$ & 0.22 & 0.11 & 0.26 & 0.10 & 0.052 & 0.86 \\
\hline & & $\mathrm{S}$ & 0.14 & 0.32 & 0.18 & 0.28 & -0.0061 & 0.99 \\
\hline & & $\mathrm{K}$ & 0.19 & 0.045 & 0.24 & 0.028 & 0.10 & 0.66 \\
\hline \multirow[t]{3}{*}{$M$} & $V / \sigma$ & $\mathrm{P}$ & 0.16 & 0.28 & 0.078 & 0.64 & 0.61 & 0.083 \\
\hline & & $\mathrm{S}$ & 0.34 & 0.019 & 0.20 & 0.22 & 0.75 & 0.026 \\
\hline & & $\mathrm{K}$ & 0.21 & 0.037 & 0.11 & 0.31 & 0.56 & 0.045 \\
\hline \multirow[t]{3}{*}{$M$} & $c$ & $\mathrm{P}$ & 0.30 & 0.0021 & 0.33 & 0.0031 & 0.25 & 0.22 \\
\hline & & $\mathrm{S}$ & 0.47 & $5.3 \times 10^{-7}$ & 0.51 & $1.5 \times 10^{-6}$ & 0.32 & 0.11 \\
\hline & & $\mathrm{K}$ & 0.34 & $3.6 \times 10^{-7}$ & 0.36 & $3.1 \times 10^{-6}$ & 0.29 & 0.042 \\
\hline \multirow[t]{3}{*}{$M$} & Age & $\mathrm{P}$ & 0.010 & 0.94 & -0.27 & 0.090 & 0.35 & 0.27 \\
\hline & & $S$ & 0.030 & 0.83 & -0.21 & 0.17 & 0.39 & 0.21 \\
\hline & & $\mathrm{K}$ & $8.8 \times 10^{-3}$ & 0.93 & -0.15 & 0.17 & 0.28 & 0.26 \\
\hline \multirow[t]{3}{*}{$M$} & Age $/ t_{\mathrm{rh}}$ & $\mathrm{P}$ & -0.31 & 0.019 & -0.32 & 0.042 & -0.29 & 0.37 \\
\hline & & $\mathrm{S}$ & -0.27 & 0.37 & -0.26 & 0.097 & -0.049 & 0.89 \\
\hline & & $\mathrm{K}$ & -0.20 & 0.036 & -0.19 & 0.081 & -0.030 & 0.95 \\
\hline \multirow[t]{3}{*}{$M$} & $\varepsilon$ & $\mathrm{P}$ & 0.17 & 0.096 & 0.065 & 0.64 & 0.28 & 0.064 \\
\hline & & $\mathrm{S}$ & 0.17 & 0.10 & 0.15 & 0.19 & 0.14 & 0.52 \\
\hline & & $\mathrm{K}$ & 0.13 & 0.076 & 0.11 & 0.16 & 0.14 & 0.34 \\
\hline
\end{tabular}



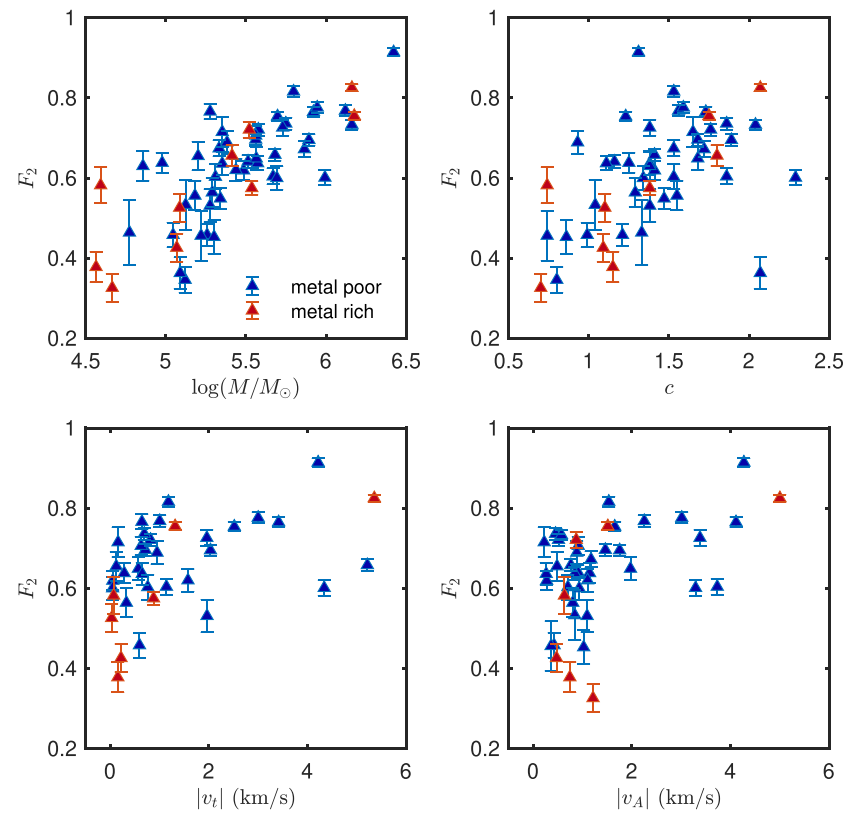

Figure A1. $F_{2}$ is plotted against the same parameters as in Fig. 1. Blue triangles are metal-poor clusters, while red triangles are metal-rich clusters.
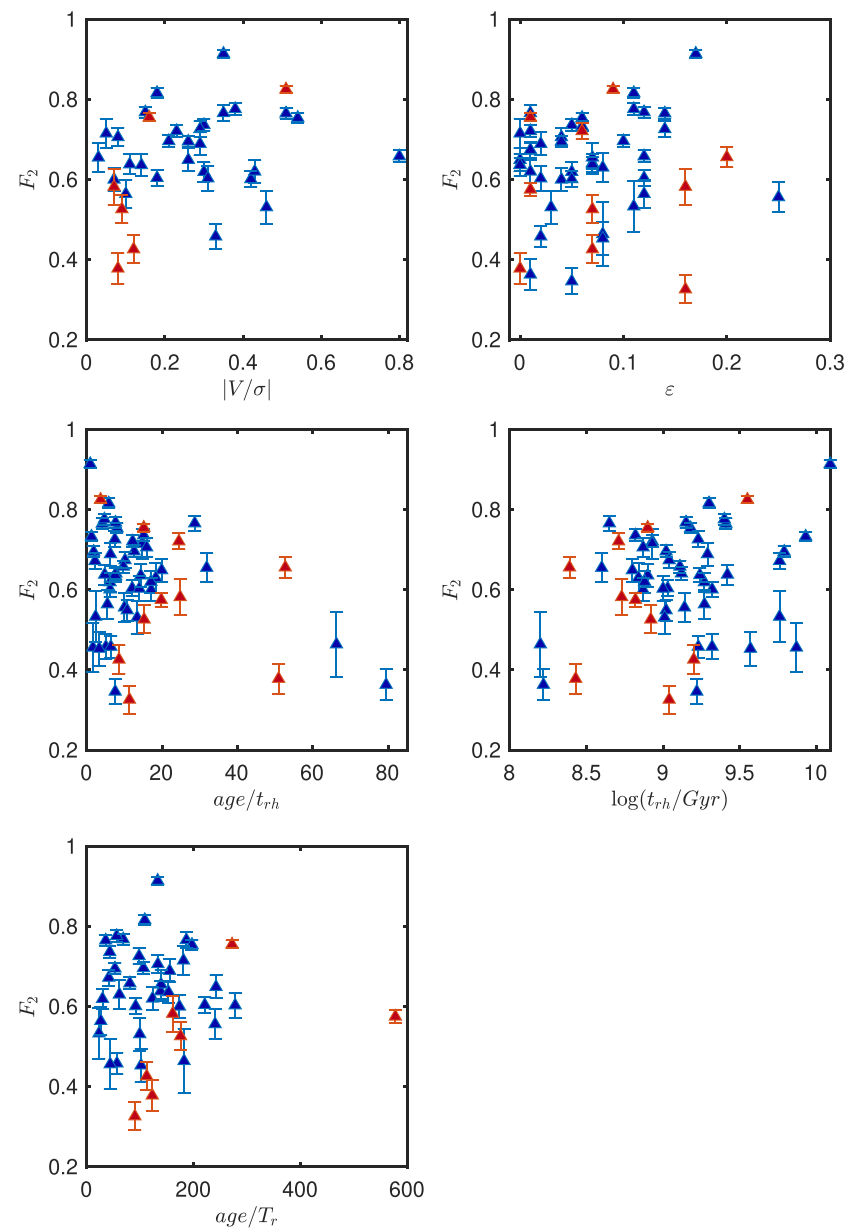

Figure A2. $F_{2}$ is plotted against the same parameters as in Fig. 2. Blue triangles are metal-poor clusters, while red triangles are metal-rich clusters.

cluster sample and the metal-poor clusters. In the case of the metalrich clusters these quantities are, instead, correlated only in the case of the Kendal statistics.

\section{APPENDIX B: CORRELATIONS AND PROJECTED ROTATIONAL VELOCITIES}

For completeness, we have also tested the projected rotational velocity, $v_{t}$, against the other internal parameters considered in this work. The results obtained considering the metallicity threshold are reported in Table B1, while the results obtained considering the mass threshold are presented in Table B2. 
Table B1. Statistical correlation coefficients (PCC) and probabilities (PP) between the projected rotational velocity, $v_{t}$, and several GC internal parameters. Parameters are reported for the entire sample of GCs, for metal-poor (MP) and metal-rich (MR) clusters. P stands for Pearson, S for Spearman, and K for Kendall.

\begin{tabular}{|c|c|c|c|c|c|c|c|c|}
\hline $\operatorname{Par}_{1}$ & $\mathrm{Par}_{2}$ & Stat & PCC & PP & $\mathrm{PCC}_{\mathrm{MP}}$ & $\mathrm{PP}_{\mathrm{MP}}$ & $\mathrm{PCC}_{\mathrm{MR}}$ & $\mathrm{PP}_{\mathrm{MR}}$ \\
\hline \multirow[t]{3}{*}{$\left|v_{t}\right|$} & \multirow[t]{3}{*}{$M$} & $\mathrm{P}$ & 0.58 & $6.9 \times 10^{-6}$ & 0.16 & 0.48 & 0.42 & 0.024 \\
\hline & & S & 0.64 & $3.6 \times 10^{-7}$ & 0.18 & 0.44 & 0.49 & 0.0070 \\
\hline & & $\mathrm{K}$ & 0.46 & $1.7 \times 10^{-6}$ & 0.13 & 0.43 & 0.33 & 0.012 \\
\hline \multirow[t]{3}{*}{$\left|v_{t}\right|$} & \multirow[t]{3}{*}{$c$} & $\mathrm{P}$ & 0.40 & 0.0068 & 0.20 & 0.43 & 0.22 & 0.28 \\
\hline & & S & 0.40 & 0.0065 & 0.23 & 0.37 & 0.091 & 0.65 \\
\hline & & K & 0.28 & 0.0071 & 0.18 & 0.32 & 0.060 & 0.68 \\
\hline \multirow[t]{3}{*}{$\left|v_{t}\right|$} & \multirow[t]{3}{*}{ Age } & $\mathrm{P}$ & 0.17 & 0.32 & 0.094 & 0.64 & 0.29 & 0.49 \\
\hline & & S & 0.14 & 0.42 & -0.011 & 0.96 & 0.29 & 0.48 \\
\hline & & $\mathrm{K}$ & 0.092 & 0.47 & -0.018 & 0.92 & 0.23 & 0.53 \\
\hline \multirow[t]{3}{*}{$\left|v_{t}\right|$} & \multirow[t]{3}{*}{ Age $/ t_{\mathrm{rh}}$} & $\mathrm{P}$ & -0.43 & 0.010 & -0.37 & 0.058 & -0.50 & 0.20 \\
\hline & & S & -0.52 & 0.0015 & -0.41 & 0.034 & -0.52 & 0.20 \\
\hline & & K & -0.37 & 0.0019 & -0.31 & 0.023 & -0.36 & 0.28 \\
\hline \multirow[t]{3}{*}{$\left|v_{t}\right|$} & \multirow[t]{3}{*}{$\varepsilon$} & $\mathrm{P}$ & 0.14 & 0.32 & -0.39 & 0.079 & 0.22 & 0.24 \\
\hline & & S & 0.14 & 0.32 & 0.18 & 0.28 & -0.0061 & 0.99 \\
\hline & & $\mathrm{K}$ & 0.10 & 0.31 & -0.24 & 0.14 & 0.16 & 0.26 \\
\hline
\end{tabular}

Table B2. Statistical correlation coefficients (PCC) and probabilities (PP) between the projected rotational velocity, $v_{t}$, and several GC internal parameters. Parameters are reported for the entire sample of GCs, for clusters more massive (HM) and less massive (LM) than $10^{5.5} \mathrm{M}_{\odot}$. P stands for Pearson, $\mathrm{S}$ for Spearman, and $\mathrm{K}$ for Kendall.

\begin{tabular}{|c|c|c|c|c|c|c|c|c|}
\hline $\operatorname{Par}_{1}$ & $\mathrm{Par}_{2}$ & Stat & PCC & PP & $\mathrm{PCC}_{\mathrm{LM}}$ & $\mathrm{PP}_{\mathrm{LM}}$ & $\mathrm{PCC}_{\mathrm{HM}}$ & $\mathrm{PP}_{\mathrm{HM}}$ \\
\hline \multirow[t]{3}{*}{$\left|v_{t}\right|$} & \multirow[t]{3}{*}{$M$} & $P$ & 0.58 & $6.9 \times 10^{-6}$ & 0.52 & $4.4 \times 10^{-4}$ & $0 / 77$ & 0.0097 \\
\hline & & S & 0.64 & $3.6 \times 10^{-7}$ & 0.58 & $7.8 \times 10^{-5}$ & 0.83 & 0.0056 \\
\hline & & K & 0.46 & $1.7 \times 10^{-6}$ & 0.40 & $2.1 \times 10^{-4}$ & 0.64 & 0.0091 \\
\hline \multirow[t]{3}{*}{$\left|v_{t}\right|$} & \multirow[t]{3}{*}{$c$} & $\mathrm{P}$ & 0.40 & 0.0068 & 0.26 & 0.13 & 0.70 & 0.022 \\
\hline & & S & 0.40 & 0.0065 & 0.25 & 0.14 & 0.82 & 0.0068 \\
\hline & & $\mathrm{K}$ & 0.28 & 0.0071 & 0.18 & 0.13 & 0.69 & 0.0047 \\
\hline \multirow[t]{3}{*}{$\left|v_{t}\right|$} & \multirow[t]{3}{*}{ Age } & $\mathrm{P}$ & 0.17 & 0.32 & 0.021 & 0.18 & -0.47 & 0.61 \\
\hline & & S & 0.14 & 0.42 & 0.54 & 0.024 & 0.039 & 0.88 \\
\hline & & $\mathrm{K}$ & 0.092 & 0.47 & 0.44 & 0.018 & 0.021 & 0.94 \\
\hline \multirow[t]{3}{*}{$\left|v_{t}\right|$} & \multirow{3}{*}{ Age $/ t_{\mathrm{rh}}$} & $\mathrm{P}$ & -0.43 & 0.010 & -0.13 & 0.61 & -0.66 & 0.0032 \\
\hline & & S & -0.52 & 0.0015 & -0.12 & 0.64 & -0.82 & $3.1 \times 10^{-5}$ \\
\hline & & K & -0.37 & 0.0019 & -0.074 & 0.72 & -0.66 & $1.8 \times 10^{-4}$ \\
\hline \multirow[t]{3}{*}{$\left|v_{t}\right|$} & \multirow[t]{3}{*}{$\varepsilon$} & $\mathrm{P}$ & 0.17 & 0.096 & 0.19 & 0.11 & 0.20 & 0.35 \\
\hline & & $\mathrm{S}$ & 0.14 & 0.32 & 0.18 & 0.28 & -0.0061 & 0.99 \\
\hline & & $\mathrm{K}$ & 0.10 & 0.30 & 0.13 & 0.26 & 0.023 & 1.0 \\
\hline
\end{tabular}

\section{APPENDIX C: AGE CORRELATIONS}

We used four different data sets to check for the existence of a relationship between $F_{2}$ and the age of the clusters (Dotter et al. 2010; Forbes \& Bridges 2010; Gratton et al. 2010; VandenBerg et al. 2013). We found a clear anticorrelation between the two quantities only when using the ages taken from Forbes \& Bridges (2010). In this case, clusters less massive than $10^{5.5} \mathrm{M}_{\odot}$ do not show any clear $F_{2}$-age correlation, while clusters more massive than the threshold display a clear trend between the two quantities when outliers are removed.

The ages from Forbes \& Bridges (2010) have been, however, collected from several heterogeneous sources and, therefore, the correlation found might be spurious. Indeed, when using the ages collected in more homogeneous ways (Dotter et al. 2010; Gratton et al. 2010; VandenBerg et al. 2013), we do not find any strong relationship between $F_{2}$ and the cluster age. This is in line with what found by Milone et al. (2017), who only detected a mild anticorrelation between the $1 \mathrm{P}$ fractions and with the ages from VandenBerg et al. (2013).
We note that when using the same clusters available with Dotter et al. (2010), Gratton et al. (2010), and VandenBerg et al. (2013), we do not find any anticorrelation, also when using the ages from Forbes \& Bridges (2010). Homogeneous and more numerous information on the ages of the clusters are necessary to determine if an anticorrelation between $F_{2}$ and the cluster age exists or not.

\section{APPENDIX D: CORRELATIONS AND BINARY FRACTIONS}

In addition to the correlations studied above, we have also investigated the existence of a trend between $F_{2}$ and the cluster binary fractions reported by Milone et al. (2012b, 2016). While Gratton et al. (2019) only considered the total binary fraction observed in the entire Hubble Space Telescope (HST) Wide Field Camera (WFC) field of view, we have looked for correlations in different radial bins, i.e. within the core radius $\left(r_{\mathrm{c}}\right)$ of the clusters, between their $r_{\mathrm{c}}$ and half-mass radius $\left(r_{\mathrm{h}}\right)$, for radii larger than $r_{\mathrm{h}}$, and in the whole WFC field. As Gratton et al. (2019), we find a very strong anticorrelation between $F_{2}$ and the binary fraction (see Fig. D1 and Table D1). 
Table D1. Statistical correlation coefficients (PCC) and probabilities (PP) between $F_{2}$ total binary fractions within the core radius $\left(r_{\mathrm{c}}\right)$, between $r_{\mathrm{c}}$ and the half-mass radius $\left(r_{\mathrm{h}}\right)$ of the clusters, outside $r_{\mathrm{h}}$, and in the full Hubble Space Telescope (HST) Wide Field Camera (WFC) field. Parameters are reported for the entire sample of GCs, for clusters more massive (HM) and less massive (LM) than $10^{5.5} \mathrm{M}_{\odot}$. P stands for Pearson, $\mathrm{S}$ for Spearman, and $\mathrm{K}$ for Kendall.

\begin{tabular}{lcccccccc}
\hline $\operatorname{Par}_{1}$ & $\mathrm{Par}_{2}$ & $\mathrm{Stat}$ & $\mathrm{PCC}$ & $\mathrm{PP}$ & $\mathrm{PCC}_{\mathrm{LM}}$ & $\mathrm{PP}_{\mathrm{LM}}$ & $\mathrm{PCC}_{\mathrm{HM}}$ & $\mathrm{PP}_{\mathrm{HM}}$ \\
\hline$F_{2}$ & $f\left(r<r_{\mathrm{c}}\right)$ & $\mathrm{P}$ & -0.39 & 0.024 & -0.12 & 0.57 & -0.89 & 0.0033 \\
& & $\mathrm{~S}$ & -0.49 & 0.0032 & -0.20 & 0.33 & -0.92 & 0.0025 \\
& & $\mathrm{~K}$ & -0.38 & 0.0017 & -0.15 & 0.28 & -0.84 & 0.0028 \\
$F_{2}$ & $f\left(r_{\mathrm{c}} \leq r<r_{\mathrm{h}}\right)$ & $\mathrm{P}$ & -0.72 & $2.8 \times 10^{-9}$ & -0.44 & 0.018 & -0.51 & 0.015 \\
& & $\mathrm{~S}$ & -0.58 & $92.6 \times 10^{-5}$ & -0.31 & 0.12 & -0.41 & 0.091 \\
& & $\mathrm{~K}$ & -0.41 & $9.7 \times 10^{-5}$ & -0.20 & 0.16 & -0.29 & 0.10 \\
$F_{2}$ & $f\left(r \geq r_{\mathrm{h}}\right)$ & $\mathrm{P}$ & -0.69 & $1.3 \times 10^{-6}$ & 0.59 & 0.0064 & -0.30 & 0.21 \\
& & $\mathrm{~S}$ & -0.73 & $1.7 \times 10^{-7}$ & 0.66 & 0.0014 & -0.40 & 0.091 \\
& & $\mathrm{~K}$ & 0.55 & $9.3 \times 10^{-7}$ & 0.49 & 0.0031 & -0.28 & 0.11 \\
$F_{2}$ & $f_{\mathrm{WFC}}$ & $\mathrm{P}$ & -0.68 & $5.6 \times 10^{-8}$ & -0.46 & 0.014 & -0.56 & 0.0068 \\
& & $\mathrm{~S}$ & -0.72 & $2.8 \times 10^{-9}$ & -0.44 & 0.018 & -0.51 & 0.015 \\
& & $\mathrm{~K}$ & -0.53 & $3.4 \times 10^{-8}$ & -0.31 & 0.022 & -0.30 & 0.049 \\
$\log (M)$ & $f_{\mathrm{WFC}}$ & $\mathrm{P}$ & -0.65 & $1.4 \times 10^{-7}$ & -0.46 & 0.014 & -0.40 & 0.053 \\
& & $\mathrm{~S}$ & -0.73 & $8.5 \times 10^{-10}$ & -0.44 & 0.018 & -0.45 & 0.026 \\
& & $\mathrm{~K}$ & -0.73 & $8.5 \times 10^{-10}$ & -0.44 & 0.018 & -0.45 & 0.026 \\
\hline
\end{tabular}
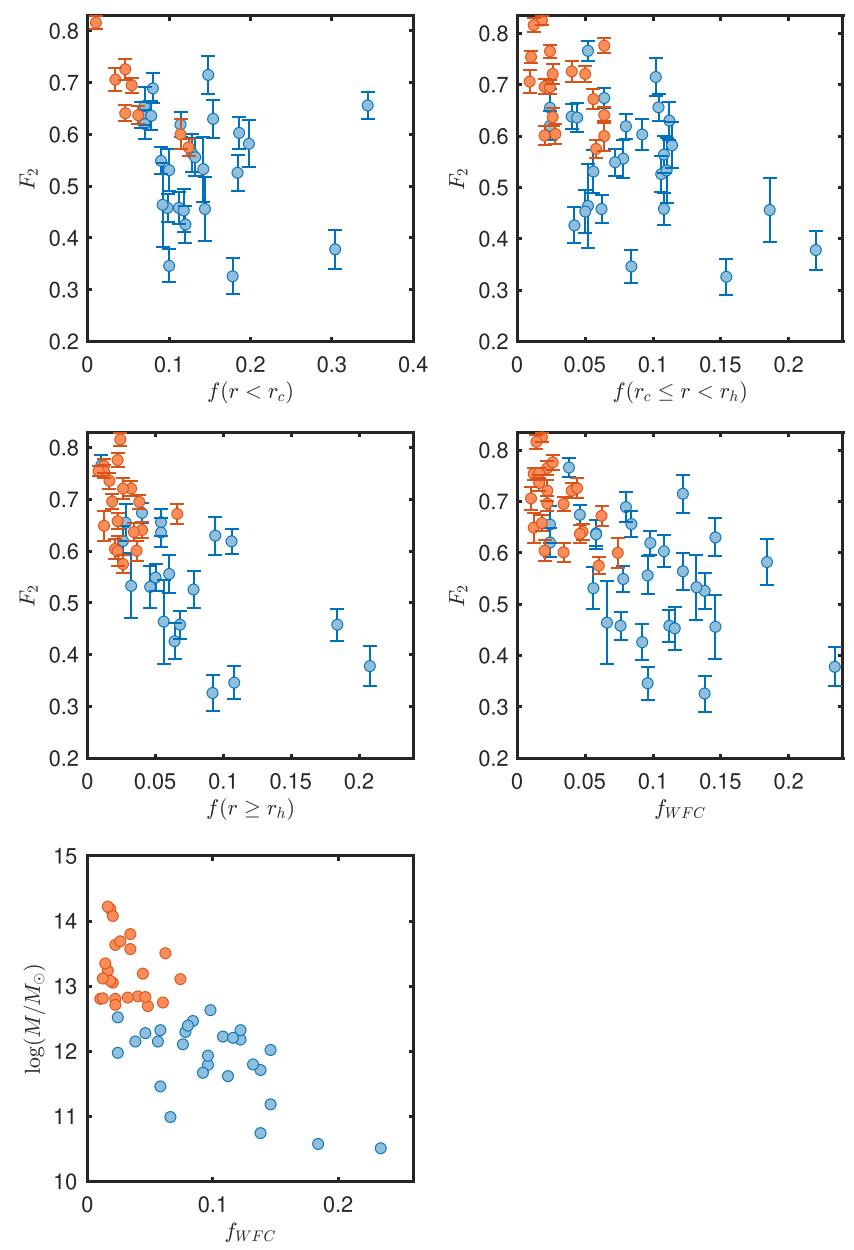

Figure D1. Going from the top to the bottom and from left to right, the fraction of $2 \mathrm{P}$ stars, $F_{2}$, is plotted against the binary fraction within the core radius, $f\left(r<r_{\mathrm{c}}\right)$, between $r_{\mathrm{c}}$ and the half-mass radius, $f\left(r_{\mathrm{c}} \leq r<r_{\mathrm{h}}\right)$, outside $r_{\mathrm{h}} f\left(r \geq r_{\mathrm{h}}\right)$, and in the full Hubble Space Telescope (HST) Wide Field Camera (WFC) field, $f_{\mathrm{WFC}}$. The bottom left-hand panel shows the logarithm of the cluster masses as a function of the binary fraction in the entire WFC field.
The anticorrelation is the tightest when considering the whole field of view $\left(f_{\mathrm{WFC}}\right)$ and its strength decreases when we consider more internal radial bins. When looking at distances smaller than $r_{\mathrm{h}}$, the anticorrelation is stronger for clusters more massive than $10^{5.5} \mathrm{M}_{\odot}$. At larger radii, as well as when we consider the entire WFC field, the anticorrelation is, instead, more pronounced for low-mass clusters.

Additionally, we observe an extremely thigh anticorrelation between the mass of the clusters and $f_{\mathrm{WFC}}$. Thus, we repeated the same regression analysis performed in Section 3.1, this time considering $f_{\mathrm{WFC}}$ as an additional parameter. The result of the regression confirms that the mass is the most important parameter $\left(p_{\text {value }}=9.8 \times 10^{-5}\right)$ driving the $2 \mathrm{P}$ phenomenon, followed by $f_{\mathrm{WFC}}\left(p_{\text {value }}=0.079\right)$. As suggested by Gratton et al. (2019), the anticorrelation between the binary fraction and $F_{2}$ could be evidence of the fact that $2 \mathrm{P}$ are born more concentrated and, therefore, with a smaller binary fraction due to a higher rate of interactions in the inner cluster regions. However, in this case, the anticorrelation should become stronger in more internal bins. The fact that we observe the exact opposite might be due to observational difficulties and to the lower number of binaries available in more collisional regions or imply that the relationship between $F_{2}$ and the binary fraction might also be mediated by the mass.

This paper has been typeset from a $\mathrm{T}_{\mathrm{E}} \mathrm{X} / \mathrm{LT} \mathrm{E} \mathrm{X}$ file prepared by the author. 